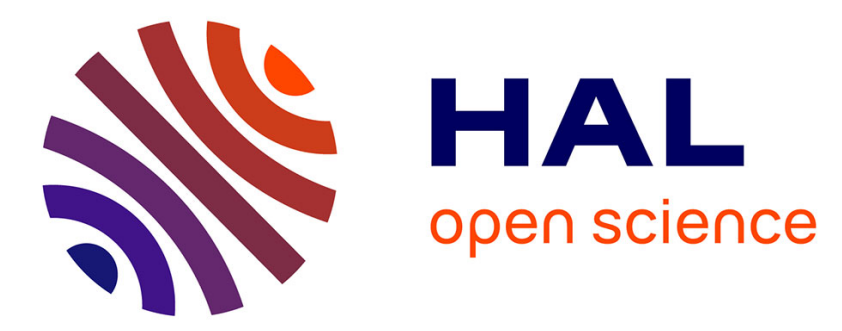

\title{
Electromagnetic waves in photonic crystals: laws of dispersion, causality and analytical properties
}

Boris Gralak, Sébastien Guenneau, Maxence Cassier, Guillaume Demésy

\section{To cite this version:}

Boris Gralak, Sébastien Guenneau, Maxence Cassier, Guillaume Demésy. Electromagnetic waves in photonic crystals: laws of dispersion, causality and analytical properties. Compendium on Electromagnetic Analysis From Electrostatics to Photonics: Fundamentals and Applications for Physicists and Engineers Volume 4: Optics and Photonics I, 2020, 10.1142/10987-vol4 . hal-03091852

\section{HAL Id: hal-03091852 \\ https://hal.science/hal-03091852}

Submitted on 31 Dec 2020

HAL is a multi-disciplinary open access archive for the deposit and dissemination of scientific research documents, whether they are published or not. The documents may come from teaching and research institutions in France or abroad, or from public or private research centers.
L'archive ouverte pluridisciplinaire HAL, est destinée au dépôt et à la diffusion de documents scientifiques de niveau recherche, publiés ou non, émanant des établissements d'enseignement et de recherche français ou étrangers, des laboratoires publics ou privés. 


\title{
Electromagnetic waves in photonic crystals: laws of dispersion, causality and analytical properties
}

\author{
Boris Gralak ${ }^{\dagger}$, Maxence Cassier, Guillaume Demésy and Sébastien Guenneau \\ Aix Marseille Univ, CNRS, Centrale Marseille \\ Institut Fresnel, Marseille, France \\ $\dagger$ boris.gralak@fresnel.fr
}

\begin{abstract}
Photonic crystals are periodic structures which prevent light propagation along one or more directions in certain frequency intervals. Their band spectrum is usually analyzed using Floquet-Bloch decomposition. This spectrum is located on the real axis, and it enters the complex plane when absorption and dispersion is considered in the dielectric permittivity of material constituents. Here, we review fundamental definition and properties of dispersion law and group velocity in photonic crystals and we illustrate them with numerical examples.
\end{abstract}

\section{Introduction}

Photonic crystals are periodic electromagnetic structures that have been originally introduced by Eli Yablonovith 11 and Sajeev John 2 in order to inhibit the spontaneous emission [3, 1] and obtain strong localization of photons 22. The original idea, based on an analogy with solid states Physics 4, was to use the periodic modulation in two or three dimensions of a lossless dielectric permittivity to open photonic bandgaps, i.e. ranges of frequencies where for which the electromagnetic radiation cannot propagate [5]. If an excited atom is embedded in such a periodic medium and if its energy level corresponds to a frequency of the bandgap, then photons cannot be radiated. Therefore photons can be strongly localized 2 and the spontaneous emission can be inhibited [3].

Hence, an important challenge of photonic crystals topic was to obtain in three-dimensions at optical wavelengths a full photonic bandgap (i.e. light is disallowed to propagate along all directions) sufficiently robust to the fabrication imperfections. The most promising structures have probably been the photonic crystals produced using colloidal suspensions [6, 7, layer-by-layer semiconductor industry technique 8, 9, 10, 11 and inverse opal synthesis 12, 13. Nevertheless, the fabrication of such three-dimensional structures remains difficult to proceed, notably in comparison with the fabrication of two-dimensional photonic crystals for which the semiconductor techniques can be directly transposed to etch membranes or slabs on substrate [14.

The ability of two-dimensional photonic crystals to forbid the propagation of the electromagnetic field has been exploited to guide light in microstructured optical fibres [15, 16, 17, 18, 19, 20, and planar structures in integrated optics [21. In photonic crystal fibers the photonic bandgap allows the guiding of light in air or vacuum, thus enabling to enhance the power of the guided light. In integrated optics, the objective was to obtain optical circuits with both reduced dimensions and a reduction of the radiation losses 22. Furthermore, the two-dimensional photonic bandgap has been used to design cavities with high quality factor 23, 24, with applications to the enhancement of the efficiency of light sources and sensors. In that case, the enhancement of the emission of photons and the electromagnetic local density of states is based on the existence of bandgaps, i.e. the absence of photonic modes for certain frequency ranges in photonic crystals.

The photonic bands themselves can be also exploited to obtain a fine control of the emission and propagation of electromagnetic waves. In addition to its enhancement, the emission of electromagnetic waves can be channelled around specific directions as soon as 
the photonic bands are restricted to the corresponding ranges of wavevectors 25, with applications to directive antennas [26]. The propagation of electromagnetic waves is governed by the photonic bands providing the dispersion law and the group velocity [27, 28. The richness of the dispersion law can lead to an enhanced dispersive effect or, conversely, to a self guiding effect [29, and to exotic refraction properties like ultra-refraction and negative refraction [30]. In particular, negative refraction from photonic crystals [31, 32, 33] can be considered as an alternative to negative index from metamaterials [34, 35, 36, since it is not spoiled by absorption.

All the aforementioned effects and applications are governed by the photonic bands and gaps which are totally determined by the relationship between the frequency $\omega$ and the wavevector $\boldsymbol{k}$, namely the dispersion law. This chapter will be devoted to this relationship including the last developments with dispersion and absorption. After the presentation of Maxwell's equations in photonic crystals in section 2, the Floquet-Bloch decomposition is introduced in section 3 It is shown that this Floquet-Bloch decomposition is a unitary transform which is specially adapted to partial differential equations with periodic coefficients since it commutes with multiplicative operator by periodic functions. Then, the dispersion law $\omega(\mathbf{k})$ is introduced in section 4 and it is shown that the group velocity $\partial_{\mathbf{k}} \omega(\mathbf{k})$ governs the propagation of the electromagnetic field. In section 5 , numerical calculations of the dispersion law are presented in the case of two-dimensional photonic crystals. In addition, the effect of the effective anisotropy on the propagation of the electromagnetic field is numerically illustrated in two-dimensional photonic crystals. In section 6 the dispersion law is extended to dispersive and absorptive photonic crystals and numerical calculation of the complex spectrum of Bloch resonances are provided for two-dimensional photonic crystals made of a Drude metal. Finally, the analytic nature of the dispersion law is discussed in section 7 .

\section{Maxwell's equations in photonic crystals}

In this chapter, different bases are used: $\left(\mathbf{e}_{1}, \mathbf{e}_{2}, \mathbf{e}_{3}\right)$ is an orthonormal basis; $\left(\mathbf{a}_{1}, \mathbf{a}_{2}, \mathbf{a}_{3}\right)$ is the basis defining the lattice associated with the photonic crystal, hence it need not be orthonormal; and $\left(\boldsymbol{K}_{1}, \boldsymbol{K}_{2}, \boldsymbol{K}_{3}\right)$ is the basis defining the reciprocal lattice. Every vector $\mathbf{x}$ in $\mathbb{R}^{3}$ (respectively in $\mathbb{C}^{3}$ ) of the physical space is described by three components $x_{1}, x_{2}$ and $x_{3}$ in $\mathbb{R}$ (respectively in $\mathbb{C}$ ).

We start with macroscopic Maxwell's equations in linear, dispersion-free dielectric media:

$$
\begin{aligned}
& \partial_{\mathbf{x}} \times \boldsymbol{E}(\mathbf{x}, t)=-\mu_{0} \partial_{t} \boldsymbol{H}(\mathbf{x}, t), \\
& \partial_{\mathbf{x}} \times \boldsymbol{H}(\mathbf{x}, t)=\varepsilon(\mathbf{x}) \partial_{t} \boldsymbol{E}(\mathbf{x}, t)+\boldsymbol{J}(\mathbf{x}, t),
\end{aligned}
$$

where $\boldsymbol{E}(\mathbf{x}, t)$ and $\boldsymbol{H}(\mathbf{x}, t)$ are the electric and magnetic fields, $\boldsymbol{J}(\mathbf{x}, t)$ is the current source density, $\partial_{\mathbf{x}} \times$ is the curl operator, $\mu_{0}$ is the vacuum permeability and $\varepsilon(\mathbf{x})$ is the dielectric permittivity. The dielectric permittivity $\varepsilon(\mathbf{x})$ in photonic crystals is generally considered as a frequency-independent function taking real and positive values greater than the one of the vacuum permittivity $\varepsilon_{0}$. Indeed, such functions can describe lossless dielectric materials which are good candidates to obtain bandgap in photonic crystals [37, while the presence of absorption implies the absence of bandgaps [38. In this chapter, the case of dispersive and absorptive permittivity is addressed in the section 6 . In the other sections, one assumes that the photonic crystal is neither dispersive nor dissipative.

Let $\mathbf{a}_{1}, \mathbf{a}_{2}$, and $\mathbf{a}_{3}$ be the linearly-independent and non-vanishing vectors of $\mathbb{R}^{3}$ defining the unit cell $V$ of the periodic photonic crystal:

$$
V=\left\{\mathbf{x}=x_{1} \mathbf{a}_{1}+x_{2} \mathbf{a}_{2}+x_{3} \mathbf{a}_{3} \mid x_{1}, x_{2}, x_{3} \in[0,1]\right\} .
$$

Then, the lattice $L$ associated with the photonic crystal is

$$
L=\left\{\mathbf{a}=p_{1} \mathbf{a}_{1}+p_{2} \mathbf{a}_{2}+p_{3} \mathbf{a}_{3} \mid p_{1}, p_{2}, p_{3} \in \mathbb{Z}\right\}
$$


and the permittivity $\varepsilon(\mathbf{x})$ determining the geometry of the crystal is invariant under the set of translations by the vectors of the lattice:

$$
\varepsilon(\mathbf{x}+\mathbf{a})=\varepsilon(\mathbf{x}), \quad \mathbf{x} \in \mathbb{R}^{3}, \mathbf{a} \in L .
$$

The basis $\left(\boldsymbol{K}_{1}, \boldsymbol{K}_{2}, \boldsymbol{K}_{3}\right)$ of the reciprocal lattice is defined such that $\boldsymbol{K}_{i} \cdot \mathbf{a}_{j}=2 \pi \delta_{i j}$ with $\delta_{i j}$ the Kronecker symbol ( $\delta_{i j}=1$ if $i=j$ and $\delta_{i j}=0$ otherwise):

$$
\boldsymbol{K}_{1}=\frac{2 \pi}{A} \mathbf{a}_{2} \times \mathbf{a}_{3}, \quad \boldsymbol{K}_{2}=\frac{2 \pi}{A} \mathbf{a}_{3} \times \mathbf{a}_{1}, \quad \boldsymbol{K}_{3}=\frac{2 \pi}{A} \mathbf{a}_{1} \times \mathbf{a}_{2},
$$

where $A=\left|\left(\mathbf{a}_{1} \times \mathbf{a}_{2}\right) \cdot \mathbf{a}_{3}\right| \neq 0$ is the volume of the unit cell $V$. Finally, the reciprocal lattice is defined by

$$
L^{*}=\left\{\boldsymbol{K}=p_{1} \boldsymbol{K}_{1}+p_{2} \boldsymbol{K}_{2}+p_{3} \boldsymbol{K}_{3} \mid p_{1}, p_{2}, p_{3} \in \mathbb{Z}\right\}
$$

and the unit cell $B$ of this reciprocal lattice, or the first Brillouin zone, is given by

$$
B=\left\{\mathbf{k}=k_{1} \boldsymbol{K}_{1}+k_{2} \boldsymbol{K}_{2}+k_{3} \boldsymbol{K}_{3} \mid k_{1}, k_{2}, k_{3} \in[-1 / 2,1 / 2]\right\} .
$$

The following purely electromagnetic quantity, corresponding to the electromagnetic energy if the field is in vacuum, is assumed to be finite for all time $t$ :

$$
\mathcal{E}(t)=\frac{1}{2} \int_{\mathbb{R}^{3}} d \mathbf{x}\left[\varepsilon_{0} \boldsymbol{E}(\mathbf{x}, t)^{2}+\mu_{0} \boldsymbol{H}(\mathbf{x}, t)^{2}\right] \quad<\infty .
$$

This assumption implies that the electromagnetic fields $\boldsymbol{E}(\mathbf{x}, t)$ and $\boldsymbol{H}(\mathbf{x}, t)$ are square integrable functions of the position $\mathbf{x}$ with well-defined Fourier transforms $\widehat{\boldsymbol{E}}(\mathbf{k}, t)$ and $\widehat{\boldsymbol{H}}(\mathbf{k}, t)$.

\section{The Floquet-Bloch decomposition}

The Floquet-Bloch decomposition is a unitary transform adapted to partial derivative equations with periodic coefficients [39, 40]. This decomposition exploits the invariance of the equations under the group of symmetries formed by the set of translations by the vectors a in the lattice $L$ (3). In this chapter, it is shown that the Maxwell's equations (1) with periodic permittivity $\varepsilon(\mathbf{x})$ are equivalent to a family of similar equations indexed by the Bloch wavevector $\mathbf{k}$ and restricted to the unit cell $V$. Also, the electromagnetic fields $\boldsymbol{E}(\mathbf{x}, t)$ and $\boldsymbol{H}(\mathbf{x}, t)$ can be uniquely defined as the superposition of Bloch waves indexed by the Bloch wavevector $\mathbf{k}$ spanning the Brillouin zone $B$. The arguments supporting these results, based on the Fourier transform and Fourier series are briefly presented and then are concluded by a summary on the Floquet-Bloch decomposition.

From the Fourier analysis to the Floquet-Bloch decomposition. The Fourier transforms $\widehat{\boldsymbol{E}}(\mathbf{k}, t)$ and $\widehat{\boldsymbol{H}}(\mathbf{k}, t)$ can be related to the fields $\boldsymbol{E}(\mathbf{x}, t)$ and $\boldsymbol{H}(\mathbf{x}, t)$ with the following integral expressions: for $\boldsymbol{F}=\boldsymbol{E}, \boldsymbol{H}$

$$
\widehat{\boldsymbol{F}}(\mathbf{k}, t)=\int_{\mathbb{R}^{3}} d \mathbf{x} \exp [-i \mathbf{k} \cdot \mathbf{x}] \boldsymbol{F}(\mathbf{x}, t),
$$

and, conversely,

$$
\boldsymbol{F}(\mathbf{x}, t)=\frac{1}{(2 \pi)^{3}} \int_{\mathbb{R}^{3}} d \mathbf{k} \exp [i \mathbf{k} \cdot \mathbf{x}] \widehat{\boldsymbol{F}}(\mathbf{k}, t) .
$$

This last expression can be decomposed using the reciprocal lattice:

$$
\boldsymbol{F}(\mathbf{x}, t)=\frac{1}{(2 \pi)^{3}} \int_{B} d \mathbf{k} \sum_{\boldsymbol{K} \in L^{*}} \exp [i(\mathbf{k}+\boldsymbol{K}) \cdot \mathbf{x}] \widehat{\boldsymbol{F}}(\mathbf{k}+\boldsymbol{K}, t) .
$$

Let $\boldsymbol{F}_{\#}(\mathbf{x}, \mathbf{k}, t)$ denote the series under the integral:

$$
\boldsymbol{F}_{\#}(\mathbf{x}, \mathbf{k}, t)=\frac{1}{(2 \pi)^{3}} \sum_{\boldsymbol{K} \in L^{*}} \exp [i(\mathbf{k}+\boldsymbol{K}) \cdot \mathbf{x}] \widehat{\boldsymbol{F}}(\mathbf{k}+\boldsymbol{K}, t) .
$$


This function appears to be periodic of $\mathbf{k}$, i.e. invariant under translations of vectors $\boldsymbol{K}$ in the reciprocal lattice $L^{*}$. Hence, it can be expanded as the Fourier series

$$
\boldsymbol{F}_{\#}(\mathbf{x}, \mathbf{k}, t)=\sum_{\mathbf{a} \in L} \exp [-i \mathbf{k} \cdot \mathbf{a}] \frac{A}{(2 \pi)^{3}} \int_{B} d \mathbf{k}^{\prime} \exp \left[i \mathbf{k}^{\prime} \cdot \mathbf{a}\right] \boldsymbol{F}_{\#}\left(\mathbf{x}, \mathbf{k}^{\prime}, t\right)
$$

where it has been used that $(2 \pi)^{3} / A=\left|\left(\boldsymbol{K}_{1} \times \boldsymbol{K}_{2}\right) \cdot \boldsymbol{K}_{3}\right|$ is the volume of the first Brillouin zone $B$. Replacing $\boldsymbol{F}_{\#}\left(\mathbf{x}, \mathbf{k}^{\prime}, t\right)$ by its series expression $(12)$, the coefficients of the Fourier series become (up to the factor $A /(2 \pi)^{3}$ )

$$
\begin{aligned}
\int_{B} d \mathbf{k}^{\prime} \exp \left[i \mathbf{k}^{\prime}\right. & \cdot \mathbf{a}] \boldsymbol{F}_{\#}\left(\mathbf{x}, \mathbf{k}^{\prime}, t\right) \\
& =\int_{B} d \mathbf{k}^{\prime} \exp \left[i \mathbf{k}^{\prime} \cdot \mathbf{a}\right] \frac{1}{(2 \pi)^{3}} \sum_{\boldsymbol{K} \in L^{*}} \exp \left[i\left(\mathbf{k}^{\prime}+\boldsymbol{K}\right) \cdot \mathbf{x}\right] \widehat{\boldsymbol{F}}\left(\mathbf{k}^{\prime}+\boldsymbol{K}, t\right) \\
& =\int_{B} d \mathbf{k}^{\prime} \frac{1}{(2 \pi)^{3}} \sum_{\boldsymbol{K} \in L^{*}} \exp \left[i\left(\mathbf{k}^{\prime}+\boldsymbol{K}\right) \cdot(\mathbf{x}+\mathbf{a})\right] \widehat{\boldsymbol{F}}\left(\mathbf{k}^{\prime}+\boldsymbol{K}, t\right)
\end{aligned}
$$

where we used that $\exp [i \boldsymbol{K} \cdot \mathbf{a}]=1$. From $(11)$, the coefficients in $(13-(14)$ are

$$
\int_{B} d \mathbf{k}^{\prime} \exp \left[i \mathbf{k}^{\prime} \cdot \mathbf{a}\right] \boldsymbol{F}_{\#}\left(\mathbf{x}, \mathbf{k}^{\prime}, t\right)=\boldsymbol{F}(\mathbf{x}+\mathbf{a}, t) \text {. }
$$

Hence, combining (11), 12), (13) and (15), we deduce that the function $F(\mathbf{x}, t)$ can be written as the superposition over the the first Brillouin zone

$$
\boldsymbol{F}(\mathbf{x}, t)=\int_{B} d \mathbf{k} \boldsymbol{F}_{\#}(\mathbf{x}, \mathbf{k}, t)
$$

with components

$$
\boldsymbol{F}_{\#}(\mathbf{x}, \mathbf{k}, t)=\frac{A}{(2 \pi)^{3}} \sum_{\mathbf{a} \in L} \exp [-i \mathbf{k} \cdot \mathbf{a}] \boldsymbol{F}(\mathbf{x}+\mathbf{a}, t) .
$$

The linear transformation 17$)$ that defines $\boldsymbol{F}_{\#}(\mathbf{x}, \mathbf{k}, t)$ in term of $\boldsymbol{F}(\mathbf{x}, t)$ is usually referred in the literature 40, as the Floquet-Bloch transform of the function $\boldsymbol{F}(\mathbf{x}, t)$, whereas the equation (16) gives the expression of the inverse of this transform. In addition, a Parseval identity between the norms of $\boldsymbol{F}(\mathbf{x}, t)$ and $\boldsymbol{F}_{\#}(\mathbf{x}, \mathbf{k}, t)$, in the sense of square integrable functions, can be derived. Starting with the square of the norm of $\boldsymbol{F}(\mathbf{x}, t)$, the integral over the variable $\mathbf{x}$ is decomposed according to the $L$-lattice:

$$
\int_{\mathbb{R}^{3}} d \mathbf{x}|\boldsymbol{F}(\mathbf{x}, t)|^{2}=\int_{V} d \mathbf{x} \sum_{\mathbf{a} \in L}|\boldsymbol{F}(\mathbf{x}+\mathbf{a}, t)|^{2} .
$$

From (17), the function $\boldsymbol{F}_{\#}(\mathbf{x}, \mathbf{k}, t)$ is periodic of $\mathbf{k}$ and the coefficients of its series expansion are $\boldsymbol{F}(\mathbf{x}+\mathbf{a}, t)$. Hence, the square of the norm of $\boldsymbol{F}_{\#}(\mathbf{x}, \mathbf{k}, t)$ is

$$
\frac{A}{(2 \pi)^{3}} \int_{B} d \mathbf{k}\left|\boldsymbol{F}_{\#}(\mathbf{x}, \mathbf{k}, t)\right|^{2}=\sum_{\mathbf{a} \in L}|\boldsymbol{F}(\mathbf{x}+\mathbf{a}, t)|^{2}
$$

and the identity 18 becomes

$$
\int_{\mathbb{R}^{3}} d \mathbf{x}|\boldsymbol{F}(\mathbf{x}, t)|^{2}=\frac{A}{(2 \pi)^{3}} \int_{V} d \mathbf{x} \int_{B} d \mathbf{k}\left|\boldsymbol{F}_{\#}(\mathbf{x}, \mathbf{k}, t)\right|^{2}
$$

Notice that the integrals and the series have been manipulated formally without particular cautions. Rigorously, it is necessary to first consider fields decreasing "rapidly" (e.g. in the Schwartz space [41]) and then to extend the results to all finite energy (square integrable) fields using a density argument. 
Summary. The electromagnetic fields $\boldsymbol{F}(\mathbf{x}, t)$ with finite energy can be decomposed as the superposition $(16)$, over the first Brillouin zone $B$, of the Bloch waves $\boldsymbol{F}_{\#}(\mathbf{x}, \mathbf{k}, t)$ given by the equation (17). The Bloch waves $\boldsymbol{F}_{\#}(\mathbf{x}, \mathbf{k}, t)$, defined for all Bloch wavevector $\mathbf{k}$, represent the Floquet-Bloch transform of $\boldsymbol{F}(\mathbf{x}, t)$. From $(20)$, the Floquet-Bloch transform is an isometry and the decomposition 16 is unique. The Floquet-Bloch transform $\boldsymbol{F}_{\#}(\mathbf{x}, \mathbf{k}, t)$ is a $L^{*}$ periodic function with respect to the Bloch wavevector $\mathbf{k}$ while, with respect to the space variable $\mathbf{x}$, it satisfies the Bloch requirement

$$
\boldsymbol{F}_{\#}(\mathbf{x}+\mathbf{a}, \mathbf{k}, t)=\exp [i \mathbf{k} \cdot \mathbf{a}] \boldsymbol{F}_{\#}(\mathbf{x}, \mathbf{k}, t), \quad \mathbf{a} \in L .
$$

It is important to notice that the Floquet-Bloch transform of $\varepsilon(\mathbf{x}) \boldsymbol{E}(\mathbf{x}, t)$ is 17

$$
\begin{aligned}
{[\varepsilon \boldsymbol{E}]_{\#}(\mathbf{x}, \mathbf{k}, t) } & =\frac{A}{(2 \pi)^{3}} \sum_{\mathbf{a} \in L} \exp [-i \mathbf{k} \cdot \mathbf{a}] \varepsilon(\mathbf{x}+\mathbf{a}) \boldsymbol{E}(\mathbf{x}+\mathbf{a}, t) \\
& =\frac{A}{(2 \pi)^{3}} \sum_{\mathbf{a} \in L} \exp [-i \mathbf{k} \cdot \mathbf{a}] \varepsilon(\mathbf{x}) \boldsymbol{E}(\mathbf{x}+\mathbf{a}, t) \\
& =\varepsilon(\mathbf{x}) \boldsymbol{E}_{\#}(\mathbf{x}, \mathbf{k}, t)
\end{aligned}
$$

In other words, the Floquet-Bloch transform commutes with the multiplicative operator by the periodic function $\varepsilon(\mathbf{x})$. Thus the Floquet-Bloch transform appears to be particularly adapted to partial differential equations with periodic coefficients. The application of the Floquet-Bloch transform to the Maxwell's equations (1) leads to the following family of independent equations indexed by the Bloch wavevector $\mathbf{k}$ spanning the first Brillouin zone $B$ :

$$
\begin{aligned}
& \partial_{\mathbf{x}} \times \boldsymbol{E}_{\#}(\mathbf{x}, \mathbf{k}, t)=-\mu_{0} \partial_{t} \boldsymbol{H}_{\#}(\mathbf{x}, \mathbf{k}, t), \\
& \partial_{\mathbf{x}} \times \boldsymbol{H}_{\#}(\mathbf{x}, \mathbf{k}, t)=\varepsilon(\mathbf{x}) \partial_{t} \boldsymbol{E}_{\#}(\mathbf{x}, \mathbf{k}, t)+\boldsymbol{J}_{\#}(\mathbf{x}, \mathbf{k}, t) .
\end{aligned}
$$

Each equation indexed by $\mathbf{k}$ can be solved separately for fields $\boldsymbol{E}_{\#}(\mathbf{x}, \mathbf{k}, t)$ and $\boldsymbol{H}_{\#}(\mathbf{x}, \mathbf{k}, t)$ that are square integrable with respect to $\mathbf{x}$ on the unit cell $V$. Finally, the solutions $\boldsymbol{E}(\mathbf{x}, t)$ and $\boldsymbol{H}(\mathbf{x}, t)$ of the initial Maxwell's equations are retrieved performing the superposition over the first Brillouin zone:

$$
\boldsymbol{E}(\mathbf{x}, t)=\int_{B} d \mathbf{k} \boldsymbol{E}_{\#}(\mathbf{x}, \mathbf{k}, t), \quad \boldsymbol{H}(\mathbf{x}, t)=\int_{B} d \mathbf{k} \boldsymbol{H}_{\#}(\mathbf{x}, \mathbf{k}, t) .
$$

The Floquet-Bloch transform appears as the tool to decompose periodic equations into a set of equations restricted to the unit cell $V$. This transform leads also to the introduction of the Bloch wavevector $\mathbf{k}$, which is the fundamental physical conserved quantity associated with the group of symmetries formed by the set of translations of vector $\mathbf{a}$ in $L$.

\section{The dispersion law}

The Maxwell's equations (1) are invariant under any translation with respect to the time $t$. That suggests to decompose the equations with respect to the time and to consider them in the time-harmonic regime.

We start with Maxwell's equations (23) after the Floquet-Bloch decomposition and with the current source $\boldsymbol{J}_{\#}(\mathbf{x}, \mathbf{k}, t)$ set to zero. Assuming that a Fourier decomposition with respect to the time can be applied to these equations 11 the following set of equations is obtained:

$$
\begin{aligned}
& \partial_{\mathbf{x}} \times \mathbf{E}_{\#}(\mathbf{x}, \mathbf{k}, \omega)=i \omega \mu_{0} \mathbf{H}_{\#}(\mathbf{x}, \mathbf{k}, \omega), \\
& \partial_{\mathbf{x}} \times \mathbf{H}_{\#}(\mathbf{x}, \mathbf{k}, \omega)=-i \omega \varepsilon(\mathbf{x}) \mathbf{E}_{\#}(\mathbf{x}, \mathbf{k}, \omega),
\end{aligned}
$$

\footnotetext{
${ }^{1}$ It is stressed that the Fourier decomposition with respect to the time of equations [23) [or (1)] is not straightforward when the electromagnetic energy is conserved, and it has to be considered in the sense of distributions. An alternative way is to perform a Laplace transform [42] to the equations for a frequency $\omega$ with a positive imaginary part [43]. Then, the limit $\operatorname{Im}(\omega) \downarrow 0$ can be considered to define the time-harmonic Maxwell's equations (or the Helmholtz operator).
} 
where $\mathbf{E}_{\#}(\mathbf{x}, \mathbf{k}, \omega)$ and $\mathbf{H}_{\#}(\mathbf{x}, \mathbf{k}, \omega)$ are the time-harmonic electric and magnetic fields oscillating at the frequency $\omega$, with the Bloch boundary condition (21). This set of equations 25 can be expressed as the eigenvalue problem

$$
M(\mathbf{x}, \mathbf{k}) F_{\#}(\mathbf{x}, \mathbf{k}, \omega)=\omega F_{\#}(\mathbf{x}, \mathbf{k}, \omega)
$$

where

$$
F_{\#}(\mathbf{x}, \mathbf{k}, \omega)=\left[\begin{array}{c}
\mathbf{E}_{\#}(\mathbf{x}, \mathbf{k}, \omega) \\
\mathbf{H}_{\#}(\mathbf{x}, \mathbf{k}, \omega)
\end{array}\right] \exp [-i \mathbf{k} \cdot \mathbf{x}],
$$

is a square integrable periodic function of $\mathbf{x}$ on the unit cell $V$ and

$$
M(\mathbf{x}, \mathbf{k})=\left[\begin{array}{lr}
0 & i \varepsilon^{-1}(\mathbf{x})\left(\partial_{\mathbf{x}}+i \mathbf{k}\right) \times \\
-i \mu_{0}^{-1}\left(\partial_{\mathbf{x}}+i \mathbf{k}\right) \times & 0
\end{array}\right],
$$

is an operator depending on the Bloch wavevector $\mathbf{k}$. The solutions of time-harmonic Maxwell equations 25 without sources are the Bloch modes of the photonic crystal. These modes are proportional to the eigenvectors $F_{\#}(\mathbf{x}, \mathbf{k}, \omega)$ of the operator $M(\mathbf{x}, \mathbf{k})$ acting on the square integrable periodic functions of $\mathbf{x}$ on the unit cell $V$. The oscillating frequencies $\omega$ of the Bloch modes are the eigenvalues of the operator $M(\mathbf{x}, \mathbf{k})$, hence they depend on the wavevector $\mathbf{k}$. This relationship $\omega(\mathbf{k})$ defines the dispersion law.

The dispersion law $\omega(\mathbf{k})$ and the Bloch modes play a fundamental role. Indeed, the solutions of Maxwell's equations in photonic crystals can be expressed as a superposition (24) of Bloch modes. As to the dispersion law $\omega(\mathbf{k})$, it provides the relationship between the two physical invariant quantities $\omega$ and $\mathbf{k}$ resulting from the temporal and spatial symmetries. It governs the propagation of the electromagnetic field through the group velocity [27]

$$
\mathbf{v}_{g}=\left[\partial_{\mathbf{k}} \omega\right](\mathbf{k}) \text {. }
$$

This property can be justified using the following arguments. Let $\boldsymbol{X}(t)$ be the center of the electric field intensity:

$$
\boldsymbol{X}(t)=\frac{\int_{\mathbb{R}^{3}} d \mathbf{x} \mathbf{x} \boldsymbol{E}(\mathbf{x}, t)^{2}}{\int_{\mathbb{R}^{3}} d \mathbf{x} \boldsymbol{E}(\mathbf{x}, t)^{2}},
$$

where $\overline{\boldsymbol{E}(\mathbf{x}, t)}=\boldsymbol{E}(\mathbf{x}, t)$ since the time dependent field is real. Using the unitary property of the Floquet-Bloch transform, this vector becomes

$$
\boldsymbol{X}(t)=\frac{\int_{V} d \mathbf{x} \int_{B} d \mathbf{k}[\mathbf{x} \boldsymbol{E}]_{\#}(\mathbf{x}, \mathbf{k}, t) \cdot \overline{\boldsymbol{E}_{\#}(\mathbf{x}, \mathbf{k}, t)}}{\int_{V} d \mathbf{x} \int_{B} d \mathbf{k}\left|\boldsymbol{E}_{\#}(\mathbf{x}, \mathbf{k}, t)\right|^{2}}
$$

Next, the Floquet-Bloch transform of $\mathbf{x} \boldsymbol{E}(\mathbf{x}, t)$ is derived from the expression (17):

$$
[\mathbf{x} \boldsymbol{E}]_{\#}(\mathbf{x}, \mathbf{k}, t)=\sum_{\mathbf{a} \in L} \exp [-i \mathbf{k} \cdot \mathbf{a}](\mathbf{x}+\mathbf{a}) \boldsymbol{E}(\mathbf{x}+\mathbf{a}, t)=\left(\mathbf{x}+i \partial_{\mathbf{k}}\right) \boldsymbol{E}_{\#}(\mathbf{x}, \mathbf{k}, t) .
$$

Then, it is assumed that each Floquet-Bloch component $\boldsymbol{E}_{\#}(\mathbf{x}, \mathbf{k}, t)$ is made of a single timeharmonic Bloch mod $\AA^{2}$ oscillating at the frequency $\omega(\mathbf{k}): \boldsymbol{E}_{\#}(\mathbf{x}, \mathbf{k}, t)=\mathbf{E}_{\#}(\mathbf{x}, \mathbf{k}) \exp [-i \omega(\mathbf{k}) t]$ Hence the expression above becomes

$$
[\mathbf{x} \boldsymbol{E}]_{\#}(\mathbf{x}, \mathbf{k}, t)=\left\{\left[\mathbf{x}+i \partial_{\mathbf{k}}\right] \mathbf{E}_{\#}(\mathbf{x}, \mathbf{k})+\left[\partial_{\mathbf{k}} \omega\right](\mathbf{k}) t \mathbf{E}_{\#}(\mathbf{x}, \mathbf{k})\right\} \exp [-i \omega(\mathbf{k}) t],
$$

and the vector $\boldsymbol{X}(t)$ can be written

$$
\boldsymbol{X}(t)=\boldsymbol{X}_{0}+\boldsymbol{V} t
$$

\footnotetext{
2If the components $\boldsymbol{E}_{\#}(\mathbf{x}, \mathbf{k}, t)$ contain several Bloch modes, then a finite sum over the corresponding bands is obtained: note that each band has a different group velocity.
} 
where the vectors $\boldsymbol{X}_{0}$ and $\boldsymbol{V}$ are time-independent:

$$
\boldsymbol{X}_{0}=\frac{\int_{V} d \mathbf{x} \int_{B} d \mathbf{k}\left[\mathbf{x}+i \partial_{\mathbf{k}}\right] \mathbf{E}_{\#}(\mathbf{x}, \mathbf{k}) \cdot \overline{\mathbf{E}_{\#}(\mathbf{x}, \mathbf{k})}}{\int_{V} d \mathbf{x} \int_{B} d \mathbf{k}\left|\mathbf{E}_{\#}(\mathbf{x}, \mathbf{k})\right|^{2}}
$$

and

$$
\boldsymbol{V}=\frac{\int_{V} d \mathbf{x} \int_{B} d \mathbf{k}\left[\partial_{\mathbf{k}} \omega\right](\mathbf{k})\left|\mathbf{E}_{\#}(\mathbf{x}, \mathbf{k})\right|^{2}}{\int_{V} d \mathbf{x} \int_{B} d \mathbf{k}\left|\mathbf{E}_{\#}(\mathbf{x}, \mathbf{k})\right|^{2}}
$$

Thus the vector $\boldsymbol{V}$ appears as the velocity of the field intensity center. Its expression as the average of the group velocity shows that the latter governs the propagation of the electromagnetic field. Similar averaged expressions can be established for the center of the magnetic field intensity or the center of the electromagnetic field energy density. In the next section, this property of the group velocity is exploited to show the effect of the photonic crystal on the propagation of the electromagnetic field.

\section{Computation of the dispersion law and applications}

The dispersion law in photonic crystals has been investigated intensively with different numerical methods. Since the eigenvalue problem 26 is defined from the periodic operator $M(\mathbf{x}, \mathbf{k})$ acting on the Hilbert space of square integrable periodic functions of $\mathbf{x}$, the most widely used numerical method was based on the expansion of the equations into the discrete Fourier (or plane-waves) basis [4, 45, 46]. This expansion was used to predict photonic bandgap edges [47, the effect of several structural imperfections on such edges [48, the decay rate for single photon emission in infinite structures [49] and reflectivity and the inhibition of spontaneous emission for finite-thickness structures [50]. An efficient software based on this method, MPB ${ }^{3}$ for MIT Photonic Bands, has been elaborated by Steven Johnson and John Joannopoulos 51 .

In the present chapter, the finite elements software comsol 52 is used to solve eigenvalue problem 26 for transverse electromagnetic waves allowed to propagate within an infinite periodic photonic crystal (in which case the computational domain reduces to the "basic" unit cell $V$ with Floquet-Bloch boundary conditions). The software comsol is also used to solve scattering (or forced) problems for transverse electromagnetic fields radiated by a line source placed within a finite photonic crystal (in which case some perfectly matched layers are required to ensure that the boundary of the computational domain is reflectionless).
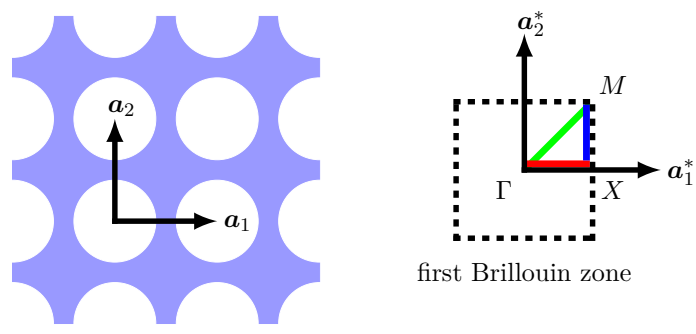

Figure 1: The considered two-dimensional photonic crystal made of circular holes drilled in a dielectric matrix. On the right the first Brillouin zone and the contour $\Gamma X M \Gamma$ of the reduced first Brillouin zone.

As an illustrative example, we consider a square photonic crystal with lattice constant $a$ and with circular air holes drilled in a matrix of permittivity $\varepsilon_{m}=11.29$ (refractive index

\footnotetext{
${ }^{3}$ https://mpb.readthedocs.io/
} 
$n=3.36)$. This permittivity value corresponds to the effective index of $s$-polarized mode in a planar waveguide made of InGaAsP $[53$. The radius of the circular holes is set to $0.455 a$, and this corresponds to the air filling ratio of 0.65 . The unit cell of the crystal is defined by the two vectors $\mathbf{a}_{1}=a \mathbf{e}_{1}$ and $\mathbf{a}_{2}=a \mathbf{e}_{2}$, and the first Brillouin zone by the vectors $\boldsymbol{K}_{1}=(2 \pi / a) \mathbf{e}_{1}$ and $\boldsymbol{K}_{2}=(2 \pi / a) \mathbf{e}_{2}$ (see Fig. 1). According to the symmetries of the unit cell, the dispersion law is represented on the path $\Gamma X M \Gamma$ in the first Brillouin zone with $\Gamma=(0,0), X=(1 / 2,0)$ and $M=(1 / 2,1 / 2)$ in the basis $\left(\boldsymbol{K}_{1}, \boldsymbol{K}_{2}\right)$. We compute the band diagrams associated with transverse electromagnetic waves propagating in the plane $\left(\mathbf{a}_{1}, \mathbf{a}_{2}\right)$. In this cylindrical case, one can split the two-dimensional spectral problem (25) into the two scalar situations identified by the $s$ - and $p$-polarizations (also referenced by, respectively, the TM and TE cases [37]). Denoting by $E_{\#} \equiv E_{\#, 3}$ and $H_{\#} \equiv H_{\#, 3}$ the components of $\boldsymbol{E}_{\#}$ and $\boldsymbol{H}_{\#}$ along the axis $\mathbf{e}_{3}$ of invariance, we look for pairs of eigenfrequencies and associated eigenfields, $\left(\omega, E_{\#}\right)$ for $s$-polarization and $\left(\omega, H_{\#}\right)$ for $p$-polarization, solutions of

$$
\begin{aligned}
& -\partial_{\mathbf{x}} \cdot \partial_{\mathbf{x}} E_{\#}(\mathbf{x}, \mathbf{k}, \omega)=\omega^{2} \mu_{0} \varepsilon(\mathbf{x}) E_{\#}(\mathbf{x}, \mathbf{k}, \omega), \\
& -\partial_{\mathbf{x}} \cdot \varepsilon^{-1}(\mathbf{x}) \partial_{\mathbf{x}} H_{\#}(\mathbf{x}, \mathbf{k}, \omega)=\omega^{2} \mu_{0} H_{\#}(\mathbf{x}, \mathbf{k}, \omega),
\end{aligned}
$$

and such that $E_{\#}$ and $H_{\#}$ satisfy the Floquet-Bloch conditions (21) on the opposite edges of the periodic unit cell $V$. Notice that, in the present two-dimensional case, the variables $\mathbf{x}$ and $\mathbf{k}$ are the two-components vectors $\left(x_{1}, x_{2}\right)$ and $\left(k_{1}, k_{2}\right)$. This problem is discretized using finite elements, and it is implemented with comsol $[52$.

$s$-polarization

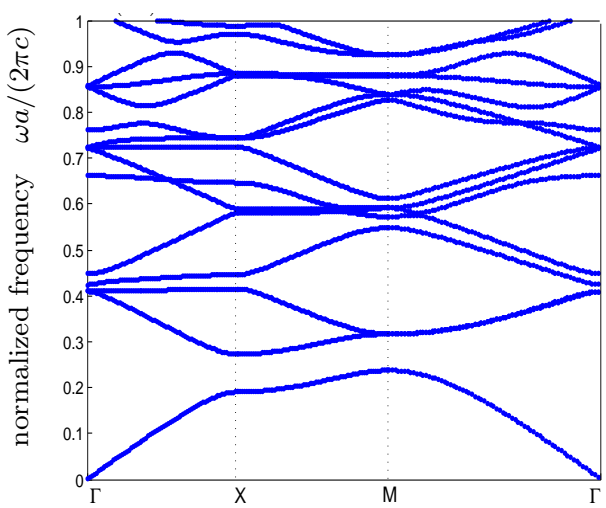

$p$-polarization

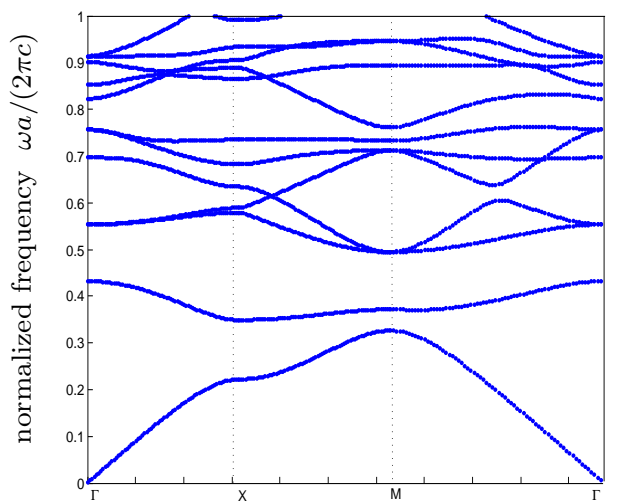

Figure 2: Dispersion diagrams in $s$ - and $p$-polarizations: normalized frequency $\omega a /(2 \pi c)$ versus Bloch wavevector $\mathbf{k}$ describing the first Brillouin zone contour $\Gamma X M \Gamma$ for transverse electromagnetic waves propagating within a doubly periodic square array of air holes of radius $0.455 a$ of center-to-center spacing $a$ in a homogeneous isotropic medium of refractive index $n=3.36$.

Figure 2 shows the dispersion diagrams in $s$ - and $p$-polarizations as the normalized frequency $\omega a /(2 \pi c)$ versus the Bloch wavevector describing the contour $\Gamma X M \Gamma, c=1 / \sqrt{\varepsilon_{0} \mu_{0}}$ being the light velocity in vacuum. These diagrams report that two-dimensional photonic bandgaps exist around $\omega a /(2 \pi c) \approx 0.26$ in $s$-polarization and around $\omega a /(2 \pi c) \approx 0.33$ or 0.48 in $p$-polarization. Thus the polarized electromagnetic fields cannot propagate at these frequencies. In addition to the bandgaps, the richness of the photonic bands $\omega(\mathbf{k})$ can be exploited in order to finely control the propagation of the electromagnetic field. The next part of this section is focused on the effect of effective anisotropy applied to source directivity.

A striking effect of directive emission of a field radiated by a line (respectively dipole line) source placed within a finite photonic crystal can be achieved at the frequency corresponding to an inflection point along the $X M$ direction of the lowest dispersion curve (known as acoustic band) in $s$ - (respectively $p$-) polarizations. Such inflection points appear along the $X M$ direction on the first band at normalized angular frequency 0.215 in left panel of Fig. 2 and at normalized frequency 0.26 in right panel of Fig. 2. It can be expected at these 
inflection points that the dispersion law restricted to the corresponding frequencies displays vanishing curvature. Indeed, these points correspond to frontier between the situation of isofrequency contours of increasing size centered about $\Gamma$ and the situation of isofrequency contours of decreasing size centered about $M$.
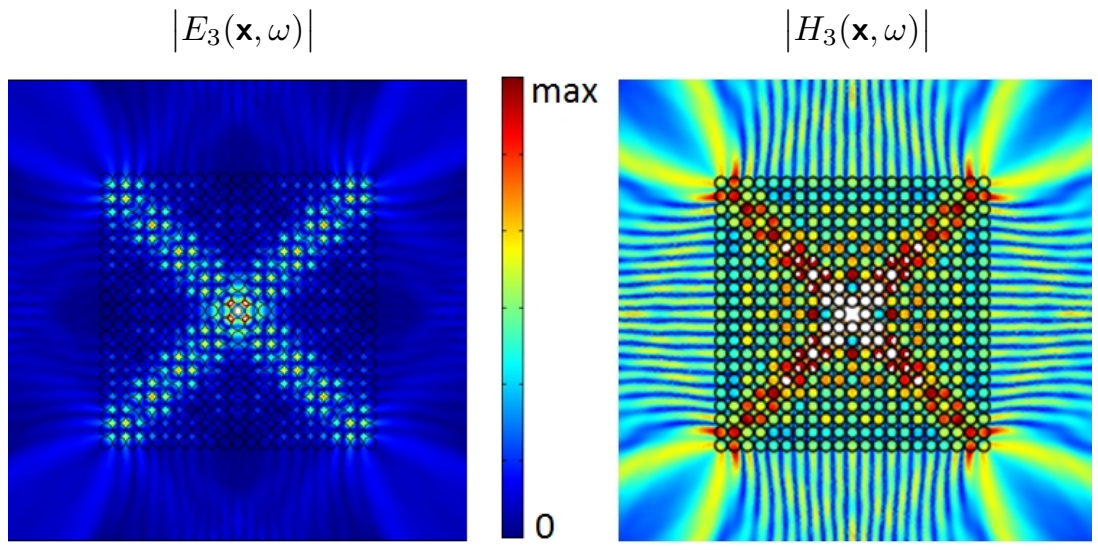

Figure 3: Effect of effective anisotropy at inflection points. Left: in $s$-polarization for a line source at normalized frequency $\omega a /(2 \pi c)=0.215$ placed at the center of the finite photonic crystal. Right: in $p$-polarization for a dipole line source at normalized frequency $\omega a /(2 \pi c)=0.26$ placed at the center of a finite photonic crystal. Note that $E_{3}$ and $H_{3}$ fields are radiated mostly along the $x=y$ and $x=-y$ directions.

To illustrate this effect, we consider a line source in $s$-polarization (respectively dipole line source in $p$-polarization) placed in the center of a finite photonic crystal with 440 air holes. When we pickup a frequency close to the inflection point on the first band in left panel of Fig. 2 (respectively in right panel of Fig. 2), we observe a striking effect in Fig. 3 whereby light emitted by the source propagates preferably along the main diagonals of the PC. Notice that this behavior can be well predicted by the so-called high-frequency homogenization theory $(\mathrm{HFH})$ [54. At these inflection points, the photonic crystal behaves like an effective medium described by an anisotropic refractive index with eigenvalues of opposite signs [55. Note that the effective medium describing the photonic crystal in Fig. 3 is actually isotropic in the low frequency regime, as the holes are circular, and achieving an anisotropy like in Fig. 3 would require extremely elongated inclusions [56].

Another interesting feature is the second band which is nearly flat for $p$-polarization in right panel of Fig. 2. If we consider the normalized frequency 0.35 for a dipole source placed in the center of the PC, we achieve a highly directive effect along the main horizontal and vertical axis in Fig. 4. Likewise, the third band is flat along $\Gamma M$ for $s$-polarization in left panel of Fig. 2, and the highly directive source emission is also shown for a line source at the normalized frequency 0.4 in Fig. 4. These infinitely anisotropic effective media in $s$ and $p$ polarizations share some common features with ultra refractive optics [25, 26.

Finally, the vital role of the interface of the photonic crystal is exemplified in Fig. 5. The situation of $p$-polarization (respectively $p$-polarization) at normalized frequency $\omega a /(2 \pi c)=$ 0.215 (respectively $\omega a /(2 \pi c)=0.26)$ is considered with the photonic crystal lattice rotated angle of $\pi / 4$ about the center of the finite crystal. Two interfaces are sliced in the direction $\left(\mathbf{a}_{1}+\mathbf{a}_{2}\right) / \sqrt{2}$. In that case, the wavevectors making a small angle with the normal to the interface correspond to the main diagonals of the photonic crystal in Fig. 3. As a result, a self-collimation along the $M \Gamma$ direction is achieved in both polarizations and a focusing effect can be observed in $s$-polarization, see Fig. 5. Note that one could implement an algorithm as described in [57] in order to reduce the impedance mismatch (and thus improve the source coupling) between crystal and surrounding medium.

Most of the features such as high-directivity and lensing effects can be captured by the high frequency homogenization. The essence of this asymptotic method is that one introduces two separate scales $\mathbf{y}$ (the macroscopic scale) and $\xi$ (the microscopic scale) and 

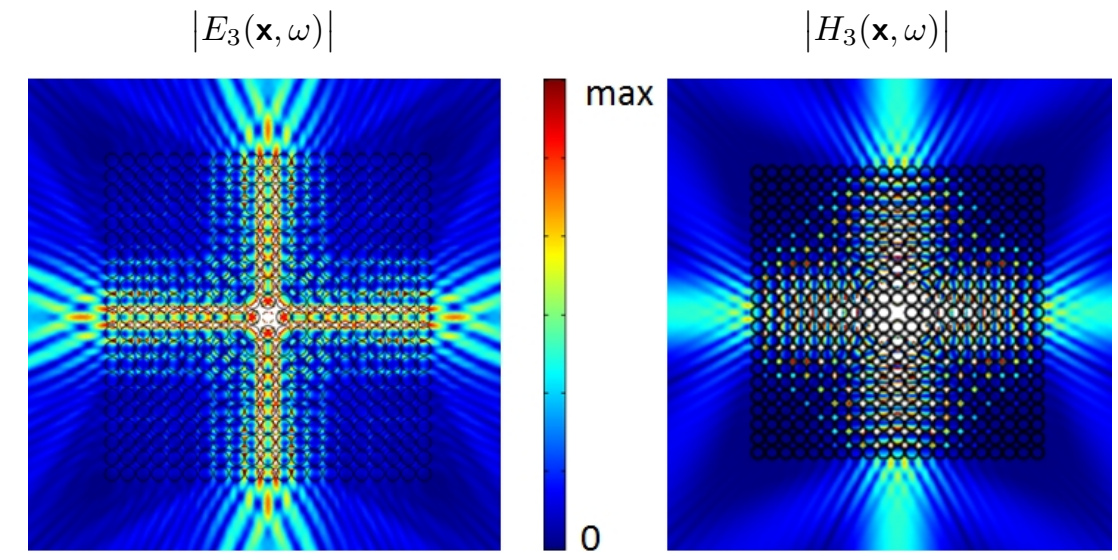

Figure 4: Effect of extreme anisotropy at near flat bands. Left : $s$-polarization for a line source at normalized frequency $\omega a /(2 \pi c)=0.4$ placed at the center of a finite photonic crystal. Right : $p$-polarization for a dipole line source at normalized frequency $\omega a /(2 \pi c)=$ 0.35 placed at the center of a finite photonic crystal. White is outside color scale. Note that $E_{3}$ and $H_{3}$ fields are radiated mostly along the $x$ and $y$ directions.

then perturbs away from high-symmetry points in the Brillouin zone and there the ansatz $H_{\#}(\mathbf{y}, \xi, \mathbf{k}, \omega)=u_{0}(\mathbf{y}, \xi)+\delta u_{1}(\mathbf{y}, \xi)+\delta^{2} u_{2}(\mathbf{y}, \xi)+\ldots$ is posed for the field $H_{\#}$ in $p$-polarization (respectively $E_{\#}$ in $s$-polarization) and $\omega^{2}=\Omega_{0}^{2}+\delta \Omega_{1}^{2}+\delta^{2} \Omega_{2}^{2}+\ldots$ for the frequency $\omega$ in (37) where $\delta$ is a small positive parameter and we have dropped the implicit dependence on $\mathbf{k}$ and $\omega$ in $u_{i}^{\prime} s$ to lighten notations. The highly oscillatory functions $u_{i}^{\prime} s$ on short scale $\xi$ get modulated by the slowly varying long scale $\mathbf{y}$. Importantly, $\Omega_{0}$ is the frequency corresponding to a standing wave $u_{0}$ associated with a Bloch vector chosen at a high symmetry point of the Brillouin zone (i.e. $\Gamma, X$ or $M)$. The $u_{i}(\mathbf{y}, \xi)$ 's adopt the boundary conditions on the edge of the cell (so periodicity or anti-periodicity as we are at a high-symmetry point). An ordered set of equations emerge indexed with their respective power of $\eta$, and are treated in turn. The leading order approximation (or homogenized field) $u_{0}$, and subsequently $u_{j}$, are computed using the standard finite element package comsol [52], although many other numerical methods could be used instead. It is then possible to replace the periodic

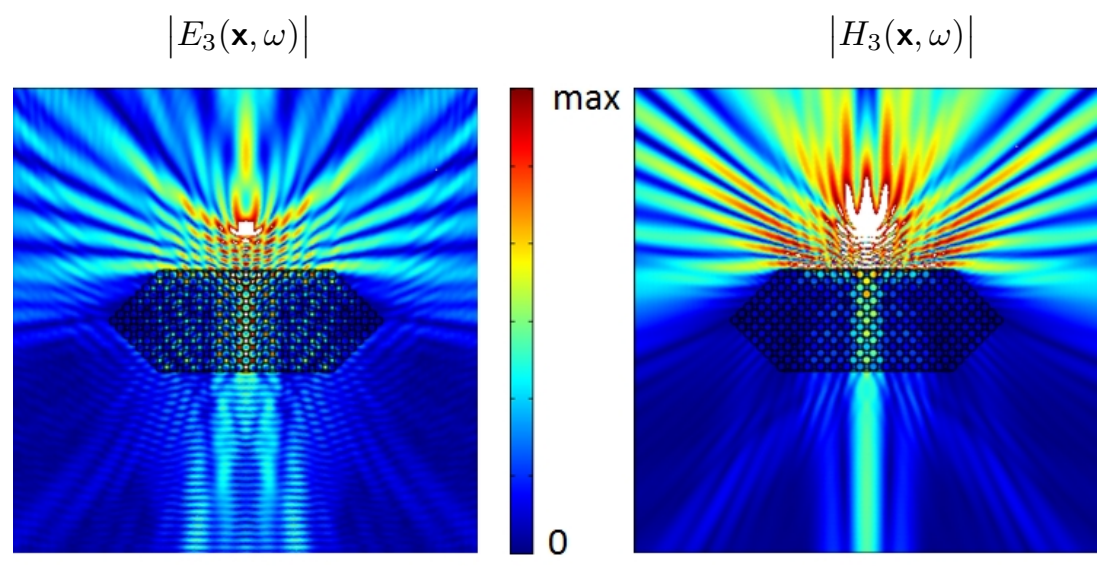

Figure 5: Effect of effective anisotropy at inflection points in a rotated photonic crystal. Left: in $s$-polarization for a line source at normalized frequency $\omega a /(2 \pi c)=0.215$ placed above a slice of the finite photonic crystal in Fig. 3 rotated through an angle pi/4 about its center. Right: in $p$-polarization for a dipole line source at normalized frequency $\omega a /(2 \pi c)=0.26$. Note that $E_{3}$ and $H_{3}$ fields are guided mostly along the $y$ direction. 
structure by an effective medium described by an anisotropic tensor. For instance, comsol computations give the following effective tensor $T_{11}=-8.6656, T_{22}=0.9209, T_{12}=T_{21}=0$ when we perturb away from the $X$ symmetry point towards the inflection point on the second band in the right panel of Fig. 2. This extremely anisotropic tensor agrees well with the directive emission of the dipole source in Fig. 4. see right panel. The theory of high frequency homogenization is introduced in [54] and some numerical illustrations for photonic crystals given in $[55]$.

Thus far, we have studied the richness of the dispersion law in dielectric photonic crystals. However, the possibility offered by the metallic materials to obtain a photonic bandgap in the visible range [58, 59] motivates use of augmented formalisms of Maxwell's equations 60, 42 for absorptive media : Metals are inherently absorptive at optical wavelengths. This is the topic of the next sections.

\section{The dispersion law in dispersive and absorptive pho- tonic crystals}

The notion of dispersive and absorptive photonic crystal appeared with the possibility offered by the metallic materials to obtain a photonic bandgap in the visible range 58, 59. The investigations on dispersion and absorption led to the definition by Adriaan Tip of the auxiliary field formalism [61, 60, which extends the Maxwell's equations to a classical evolution equation with a time-independent selfadjoint operator and thus allows the simplification of dispersion and absorption. This formalism has been used to propose a definition of photonic bandgaps in dispersive and absorptive photonic crystals 38 and to show that spontaneous emission cannot be inhibited in presence of absorption [38. Then, the computation of the dispersion law in dispersive and absorptive photonic crystals has been performed in the case of two-dimensional photonic crystals of circular [62] and square 63. rods made of a Drude metal. These preliminary calculations have been performed solving time-harmonic Maxwell's equations and using algorithms finding the complex eigenfrequencies $\omega(\mathbf{k})$ as roots of a linear system in the complex plane. A more efficient numerical method has been proposed in 64 by implementing in the numerical program a simplified version of the auxiliary field formalism. This method leads to an extension of Maxwell's equations with no dispersion but with remaining absorption. This method is now a crucial tool in the calculation of quasi-normal modes 65, 66.

In this section, the example considered in [62, 67] is revisited. The geometry is the same as in the previous section, except that the photonic crystal is made of circular rods of a Drude metal in air. The $s$-polarization is solely considered. The permittivity of the Drude metal is set to

$$
\varepsilon(\omega)=\varepsilon_{0}-\varepsilon_{0} \frac{\omega_{p}^{2}}{\omega(\omega+i \gamma)}, \quad \frac{\omega_{p} a}{2 \pi c}=1.1, \quad \frac{\gamma a}{2 \pi c}=0.05 .
$$

The computation of the eigenfrequencies is based on a version of the auxiliary field formalism that allows the linearization of the spectral problem associated with frequency dispersive materials described by Drude or Lorentz models [67. Here, the auxiliary field

$$
\boldsymbol{A}(\mathbf{x}, t)=-2 i \frac{\omega_{p}}{\sqrt{2}} \int_{-\infty}^{t} d s \exp [-\gamma(t-s)] \boldsymbol{E}(\mathbf{x}, t-s)
$$

is added to the electromagnetic field $\boldsymbol{E}(\mathbf{x}, t)$ and $\boldsymbol{H}(\mathbf{x}, t)$ to express Maxwells equations as an augmented operator independent of time (see reference [67]). A variational form of the resulting augmented system is derived and discretized using the finite element method (FEM).

One cell of the structure described in Fig. 6 is meshed using the GNU software Gmsh 68. First or second order edge elements (or Webb elements 69]) are used. The GetDP [70] GNU software allows to handle the required various basis functions handily. Very recent progress in sparse matrix eigenvalue solvers allow to tackle the discrete problem very efficiently. For 


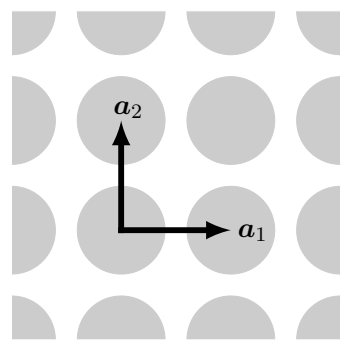

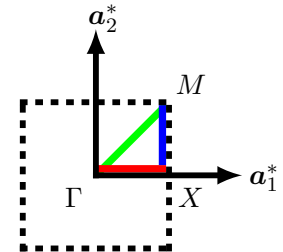

first Brillouin zone

Figure 6: The considered two-dimensional photonic crystal made of circular rods in air. On the right the first Brillouin zone and the contour $\Gamma X M \Gamma$ of the reduced first Brillouin zone: $\Gamma X$ is the red line, $X M$ is the blue line and $M \Gamma$ is the green line.

the purpose of this study, we interfaced GetDP with two particularly well suited and recent solvers of the SLEPc library 71 dedicated to solve large scale sparse eigenvalue problems.

The usual representation of the dispersion law in periodic structures provides the eigenfrequencies $\omega(\mathbf{k})$ for Bloch wavevector $\mathbf{k}$ describing the contour of the reduced first Brillouin zone. This representation is suitable for real eigenfrequencies since it maps the onedimensional contour $\Gamma X M \Gamma$ to the real axis of frequencies $\omega$. In the present case with absorption, the wavevectors $\mathbf{k}$ are mapped to complex eigenfrequencies. Hence it is relevant to describe the whole (two-dimensional) surface of the first reduced Brillouin zone and to obtain the corresponding surfaces in the complex plane of frequencies.

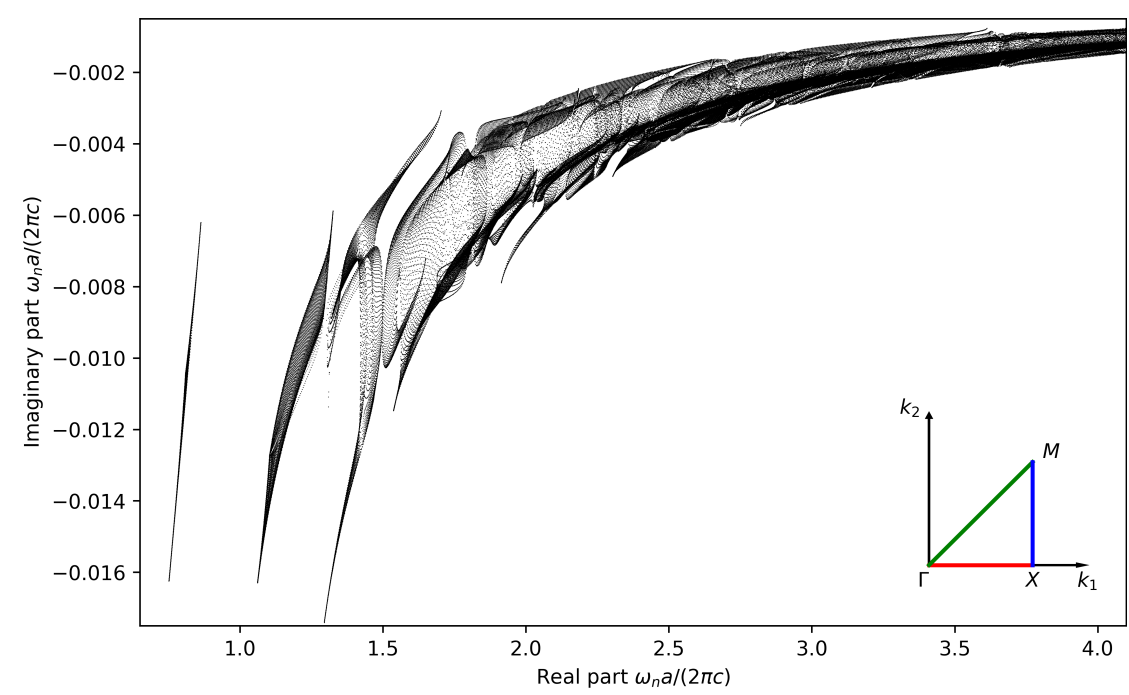

Figure 7: Complex spectrum of the normalized eigenfrequencies $\omega_{n} a /(2 \pi c)$ of photonic crystals made of and circular rods for all the wavevectors $\mathbf{k}$ in the first reduced Brillouin zone.

Figure 7 shows for $s$-polarization the whole spectrum of resonances $\omega(\mathbf{k})$ in the photonic crystal made of the Drude circular rods. The metallic nature of the Drude material around the null frequency leads to the absence of photonic bands in the range of low frequencies. Then, the first band appears well-separated of the remaining spectrum by a band around $\omega a /(2 \pi c)=1.0$, which is associated to a true photonic bandgap when the absorption parameter $\gamma$ is set to zero. The next bands appear to overlap in the complex plane to finally merge at the high frequency with the real axis. Hence the set of resonances tends toward the spectrum of the free Laplacian at high frequencies which is consistent with the behavior $\varepsilon(\omega) \rightarrow \varepsilon_{0}$ of the Drude permittivity (38) when $|\omega| \rightarrow \infty$.

Figure 8 shows the first band (left panel) and the overlapping second and third bands (right panel). The first band is a skewed triangle which can be unambiguously related to the reduced first Brillouin zone. Each edge of this triangle $\Gamma X M \Gamma$ can be clearly identified: 


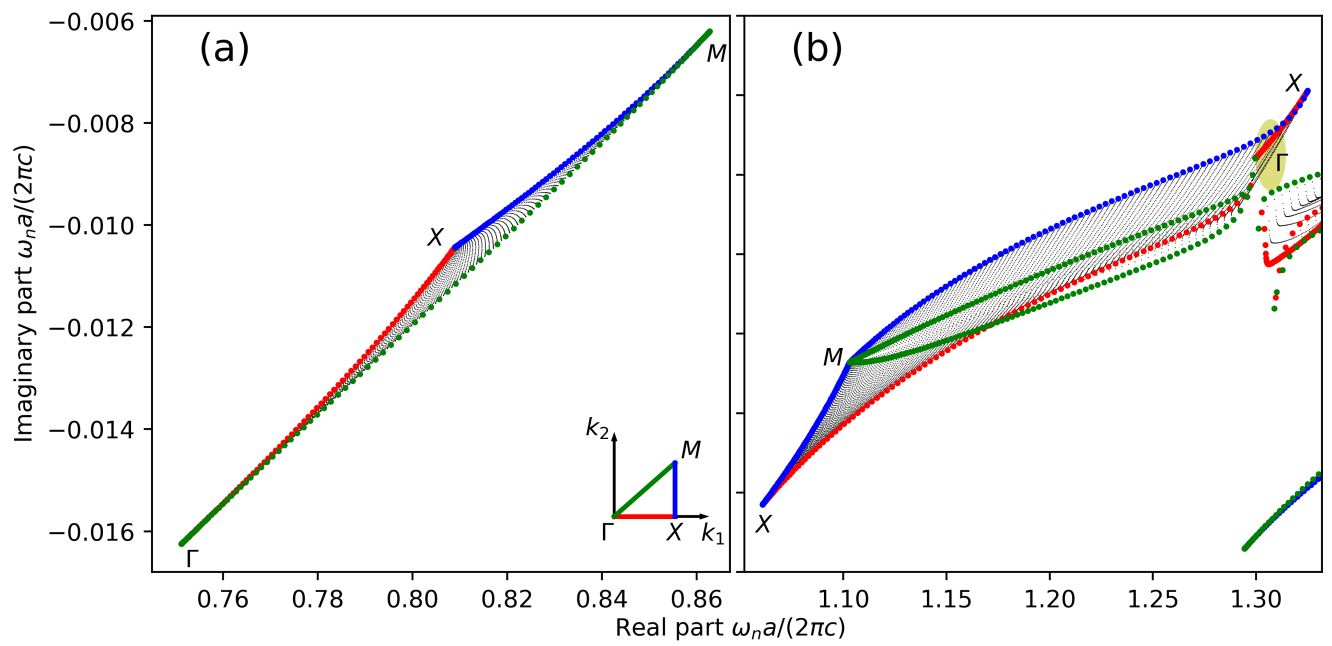

Figure 8: First band (a) and second and third bands (b) of the complex spectrum of the normalized eigenfrequencies $\omega_{n} a /(2 \pi c)$.

$\Gamma X$ is the red line, $X M$ is the blue line and $M \Gamma$ is the green line. Second and third bands overlap but can still be well-identified. However, one can remark that some resonances around the yellow annotation in Fig. 8 seem to lie outside the skewed triangles. Such a phenomenon can be clearly observed on Fig. 9 where, for instance, resonances are located between the bands 4 and 5 and seem to produce a connexion between these bands around the yellow annotation. This phenomenon, already pointed out in the literature 62, 67, is a counterexample of a widely accepted assumption in the case of non absorptive and non dispersive periodic structures: "the eigenfrequencies corresponding to the contour $\Gamma X M \Gamma$ are the extrema of eigenfrequencies of Bloch modes". According to this assumption, all the eigenfrequencies should lie inside the skewed triangle corresponding to $\Gamma X M \Gamma$. This counterexample shows the necessity to describe the whole inside of the reduced Brillouin zone to get a complete picture of the Bloch spectrum in the case of dispersive and absorptive photonic crystals.

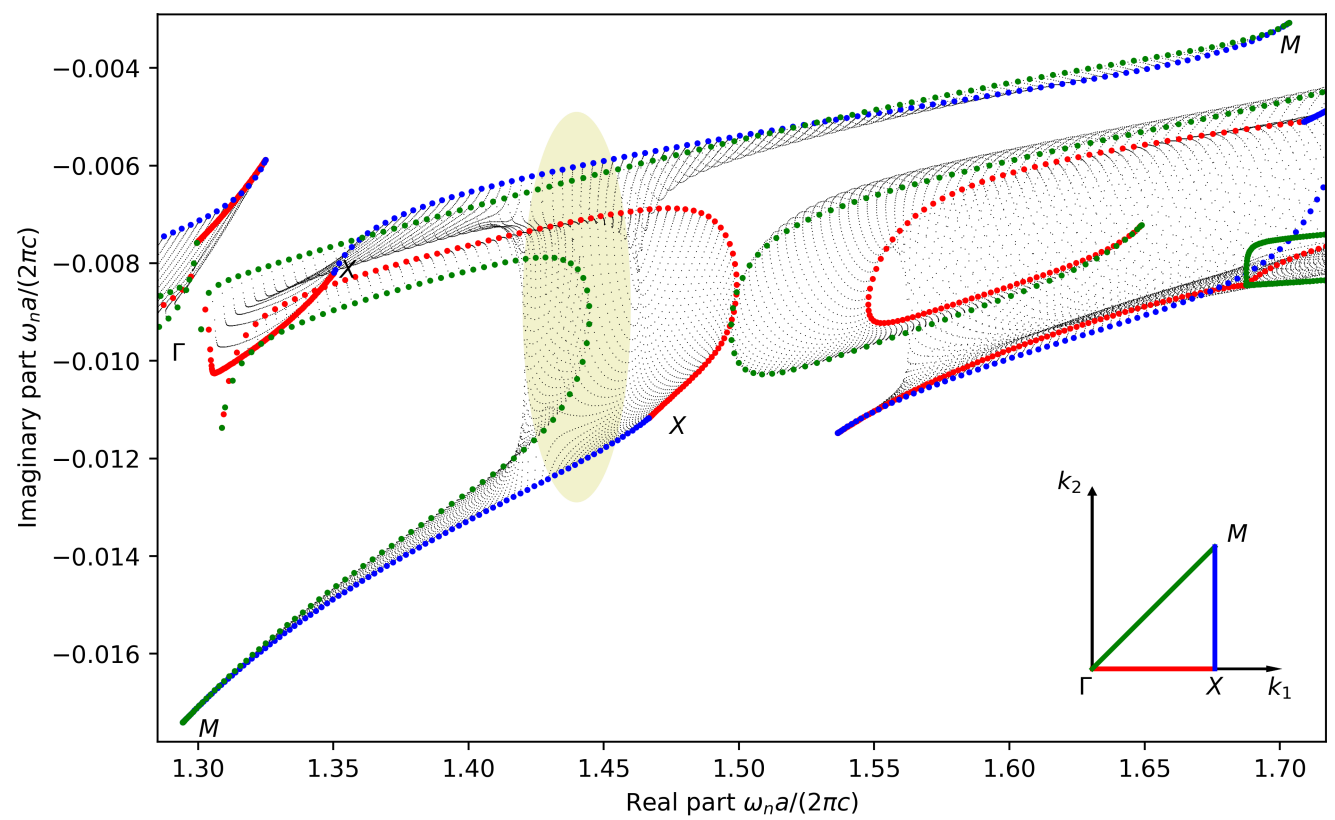

Figure 9: Bands 4, 5 and of higher order of the complex spectrum of the normalized eigenfrequencies $\omega_{n} a /(2 \pi c)$. 
The Bloch spectrum of dispersive and absorptive photonic crystals reveals a rich structure with resonances outside the contour of the reduced first Brillouin zone. As shown in reference 67, this structure of the spectrum shows in addition cups and loops with potential effects on the group velocity. These first investigations, which start from the auxiliary field formalism, represent preliminary results on the vast field of spectral theory of non-selfadjoint operators.

\section{Analytic properties of the dispersion law}

The dispersion law $\omega(\mathbf{k})$ in photonic crystal is usually considered for real Bloch wavevector $\mathbf{k}$ and frequency $\omega$. In the case of absorptive photonic crystal, it has been seen in the previous section that, for real wavevector $\mathbf{k}$, the corresponding frequencies $\omega(\mathbf{k})$ take complex values with negative imaginary part. In these two cases, the curves $\omega(\mathbf{k})$ have no global analytic structure since they are made of bands separated by gaps. However, the definition (26) of the operator $M(\mathbf{x}, \mathbf{k})$ can be extended to complex wavevectors $\mathbf{k}$. Thus its eigenvalues $\omega(\mathbf{k})$ and the dispersion law, which can be formally defined by the equation

$$
\operatorname{det}|M(\mathbf{x}, \mathbf{k})-\omega|=0
$$

can be extended to complex wavevector k. Conversely, the equation above can be extended to complex frequencies $\omega$ and the dispersion law is then defined by the roots of $(40)$ as $\mathbf{k}(\omega)$.

The aim of this section is to provide arguments supporting that the dispersion law $\mathbf{k}(\omega)$ is an analytic function of the complex frequency $\omega$ in the domain of positive imaginary parts $\operatorname{Im}(\omega)>0$. Conversely, it is reasonable to consider that the dispersion law $\omega(\mathbf{k})$ is an analytic function of $\mathbf{k}$ as soon as $\operatorname{Im}(\mathbf{k}) \neq 0$. The idea proposed in this section is to use the physical argument stating that the electromagnetic field cannot propagate faster than $c=1 / \sqrt{\varepsilon_{0} \mu_{0}}$, the light velocity in vacuum. Thus, the electromagnetic field should be strictly included in a domain defined by a condition similar to $|\mathbf{x}|<$ ct. Then, a Paley-Wiener argument 41. could be used to show the equivalence between the compact support of the electromagnetic field related to $|\mathbf{x}|<c t$ and the analytic properties with respect to the variables $\mathbf{k}$ and $\omega$ in the corresponding Fourier space. These arguments will be developed in the next part of this section. Finally, it is mentioned that a rigorous derivation in the two-dimensional case can be found in reference 72 .

The analytic properties of the solution of Maxwell's equations. The time-dependent Maxwell equations (1) are considered with the current source density $\boldsymbol{J}(\mathbf{x}, t)$ switched on at the initial time $t=0$ and homogeneous in the ball of radius $c t_{0}>0$ and centered at the origin $\mathbf{x}=0$ :

$$
\boldsymbol{J}(\mathbf{x}, t)=\boldsymbol{J}_{0} \theta\left(c t_{0}-|\mathbf{x}|\right) \theta(t) \sin \left[\omega_{s} t\right],
$$

where $\boldsymbol{J}_{0}$ is a constant vector in $\mathbb{R}^{3}, \omega_{s}$ is the oscillating frequency of the source and $\theta(t)$ is the step function: $\theta(t)=0$ for $t<0$ and $\theta(t)=1$ for $t \geq 0$. Let $G(\mathbf{x}, t)$ be the electric field radiated by this electromagnetic source : from Maxwell's equations (1),

$$
\mu_{0} \varepsilon(\mathbf{x}) \partial_{t}^{2} G(\mathbf{x}, t)+\partial_{\mathbf{x}} \times \partial_{\mathbf{x}} \times G(\mathbf{x}, t)=-\mu_{0} \boldsymbol{J}_{0} \theta\left(c t_{0}-|\mathbf{x}|\right) \theta(t) \omega_{s} \cos \left[\omega_{s} t\right]
$$

According to the causality principle, the field $G(\mathbf{x}, t)$ radiated by this source switched on at $t=0$ must vanish for all negative times. Moreover, since the electromagnetic field cannot propagate faster than the light velocity in vacuum $c=1 / \sqrt{\varepsilon_{0} \mu_{0}}$, we must have for $t>0$

$$
c\left(t+t_{0}\right)<|\mathbf{x}| \Longrightarrow G(\mathbf{x}, t)=0 \text {. }
$$

First, the analytic property with respect to the complex frequency $\omega$ is derived. Since the function $G(\mathbf{x}, t)$ vanishes for all times $t<0$, the Laplace transform

$$
\widetilde{G}(\mathbf{x}, \omega)=\int_{0}^{\infty} d t \exp [i \omega t] G(\mathbf{x}, t)
$$


is well-defined for all complex frequency with positive imaginary part $\operatorname{Im}(\omega)$. Indeed, the field $G(\mathbf{x}, t)$ cannot increase faster than linearly with time 4 , while the exponential factor $\exp [i \omega t]$ introduces the exponential decrease $\exp [-\operatorname{Im}(\omega) t]$. The integral $(44)$ as well as all its derivatives with respect to the frequency:

$$
\frac{d^{p} \widetilde{G}}{d \omega^{p}}(\mathbf{x}, \omega)=\int_{0}^{\infty} d t(i \omega)^{p} \exp [i \omega t] G(\mathbf{x}, t), \quad \forall p \in \mathbb{N},
$$

are well-defined since the integrated function are integrable for $\omega$ in the upper half-plane. Thus the Laplace transform $\widetilde{G}(\mathbf{x}, \omega)$ is analytic with respect to the complex frequency $\omega$ in the domain $\operatorname{Im}(\omega)>0$. The initial field field $G(\mathbf{x}, t)$ can be retrieved using the inverse Laplace transform:

$$
G(\mathbf{x}, t)=\frac{1}{2 \pi} \int_{R_{\eta}} d t \exp [-i \omega t] \widetilde{G}(\mathbf{x}, \omega),
$$

where $R_{\eta}$ is the line parallel to the real axis of complex frequencies with the imaginary part set to $\eta>0$,

$$
R_{\eta}=\{\omega \in \mathbb{C} \mid \operatorname{Im}(\omega)=\eta\} .
$$

For negative times $t$, the integral 46 can be calculated by closing the contour integral with a semi circle in the upper half plane of complex frequencies. Therefore, the analytic property of the Laplace transformed $\widetilde{G}(\mathbf{x}, \omega)$ in the domain $\operatorname{Im}(\omega)>0$ implies that $G(\mathbf{x}, t)=0$ for $t<0$. Hence the following equivalence has been established:

$$
G(\mathbf{x}, t)=0 \quad \forall t<0 \Longleftrightarrow \widetilde{G}(\mathbf{x}, \omega) \text { analytic } \forall \omega \text { with } \operatorname{Im}(\omega)>0 .
$$

Second, the analytic property with respect to the complex vector $\mathbf{k}$ is investigated. Assuming first a Fourier transform of the field with respect to the space variable $\mathbf{x}$, and then a Laplace transform (44), yields

$$
\begin{aligned}
\widetilde{\widetilde{G}}(\mathbf{k}, \omega) & =\int_{0}^{\infty} d t \exp [i \omega t] \int_{\mathbb{R}^{3}} d \mathbf{x} \exp [-i \mathbf{k} \cdot \mathbf{x}] G(\mathbf{x}, t) \\
& =\int_{0}^{\infty} d t \int_{|\mathbf{x}|<c\left(t+t_{0}\right)} d \mathbf{x} \exp [i(\omega t-\mathbf{k} \cdot \mathbf{x})] G(\mathbf{x}, t),
\end{aligned}
$$

where the causality condition (43) has been used. For frequency $\omega$ with positive imaginary part, the exponential function under the integral can be bounded by

$$
\begin{aligned}
|\exp [i(\omega t-\mathbf{k} \cdot \mathbf{x})]| & \leq \exp [-\operatorname{Im}(\omega) t+|\operatorname{Im}(\mathbf{k}) \cdot \mathbf{x}|] \\
& \leq \exp \left[-\operatorname{Im}(\omega) t+|\operatorname{Im}(\mathbf{k})| c\left(t+t_{0}\right)\right] \\
& \leq \exp [-\{\operatorname{Im}(\omega)-c|\operatorname{Im}(\mathbf{k})|\} t] \exp \left[c|\operatorname{Im}(\mathbf{k})| t_{0}\right]
\end{aligned}
$$

Thus the integral expression 490 of $\widetilde{G}(\mathbf{k}, \omega)$ is well-defined if $\operatorname{Im}(\omega)-c|\operatorname{Im}(\mathbf{k})|>0$, and that remains true for all the derivatives of $\widetilde{G}(\mathbf{k}, \omega)$ with respect to $\omega$ and to (the components of) k. The space-time extension of 48 is then

$$
G(\mathbf{x}, t)=0 \quad \text { if } \quad c\left(t+t_{0}\right)<|\mathbf{x}| \Longleftrightarrow \widetilde{\widetilde{G}}(\mathbf{k}, \omega) \text { analytic if } \operatorname{Im}(\omega)>c|\operatorname{Im}(\mathbf{k})| .
$$

This relationship shows the equivalence between the causality principle and the analytic properties in the Fourier-Laplace space. Next, the objective is to transfer some analytic properties from the solution $\widetilde{G}(\mathbf{k}, \omega)$ to the dispersion law $\omega(\mathbf{k})$.

\footnotetext{
${ }^{4}$ Strictly speaking, the electromagnetic energy cannot increase faster than linearly with time since the equation 42 can be written as an evolution equation (satisfying the Hille-Yosida theorem) which involves a dissipative operator with an excitation whose energy is uniformly bounded in time 73 . In practice, it is reasonable to consider that it remains true for $G(\mathbf{x}, t)$ in standard physical situations, in particular with the source 41 .
} 
The analytic properties of the dispersion law. The following section gives a reasoning that enlightens the analytic properties of the dispersion law with respect to frequency in the upper-half plane but it is not strictly speaking a rigorous mathematical proof. To our knowledge, showing the analytic regularity of the dispersion law is still an open problem in mathematics for three-dimensional photonic crystals, but it has been established for the two-dimensional case in 72 .

The solution $G(\mathbf{x}, t)$ of Maxwell's equations can be retrieved from the inverse FourierLaplace transform applied to 490 :

$$
G(\mathbf{x}, t)=\frac{1}{(2 \pi)^{4}} \int_{R_{\eta}} d \omega \int_{\mathbb{R}^{3}} d \mathbf{k} \exp [-i \omega t] \exp [i \mathbf{k} \cdot \mathbf{x}] \widetilde{G}(\mathbf{k}, \omega)
$$

Let $k=|\mathbf{k}|=\sqrt{\mathbf{k} \cdot \mathbf{k}}$ be the modulus of $\mathbf{k}$ and $\mathbf{e}_{\mathbf{k}}=\mathbf{k} / k$ the unit vector pointing in the direction of $\mathbf{k}$. The integral over the wavevectors $\mathbf{k}$ is performed with respect to the wavenumber $k$ and their directions $\mathbf{e}_{\mathbf{k}}$ on the unit sphere $S$ :

$$
G(\mathbf{x}, t)=\frac{1}{(2 \pi)^{4}} \int_{R_{\eta}} d \omega \int_{S} d \mathbf{e}_{\mathbf{k}} \int_{0}^{\infty} d k k^{2} \exp [-i \omega t] \exp \left[i k \mathbf{e}_{\mathbf{k}} \cdot \mathbf{x}\right] \widetilde{G}(\mathbf{k}, \omega) .
$$

Let $S_{\mathbf{x}}^{+}$be the hemisphere defined by

$$
S_{\mathbf{x}}^{+}=\left\{\mathbf{e}_{\mathbf{k}} \in S \mid \mathbf{e}_{\mathbf{k}} \cdot \mathbf{x}>0\right\} .
$$

Then, the expression $(53)$ can be written

$$
G(\mathbf{x}, t)=\frac{1}{(2 \pi)^{4}} \int_{R_{\eta}} d \omega \exp [-i \omega t] \int_{S_{\mathbf{x}}^{+}} d \mathbf{e}_{\mathbf{k}} \int_{\mathbb{R}} d k k^{2} \exp \left[i k \mathbf{e}_{\mathbf{k}} \cdot \mathbf{x}\right] \widetilde{G}(\mathbf{k}, \omega) .
$$
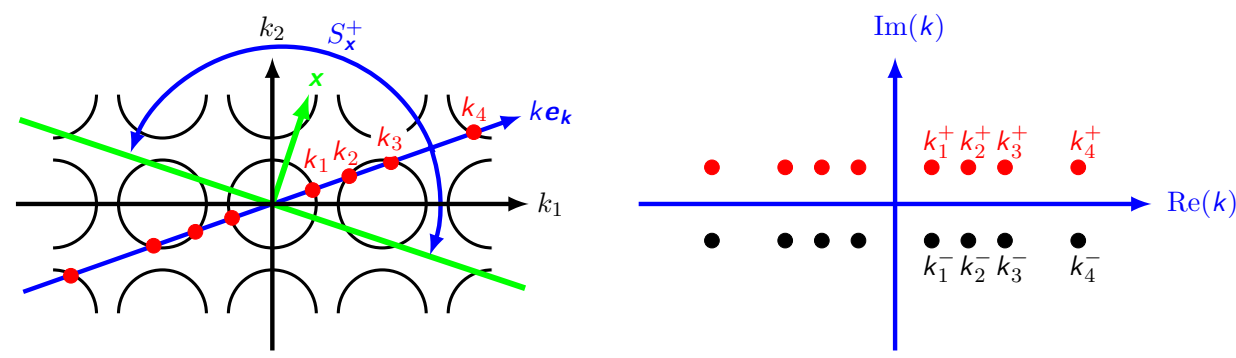

Figure 10: A representation of the poles $k_{p}^{+}=k_{p}^{+}\left(\omega, \mathbf{e}_{\mathbf{k}}\right)$. Left: the dispersion law for a real frequency represented as periodic circles. For the direction $\mathbf{e}_{\mathbf{k}}$, the wavevector $\mathbf{k}_{\mathbf{k}}$ meets a discrete infinite number of Bloch wavevectors. When the direction $\mathbf{e}_{\mathbf{k}}$ describes all the hemisphere $S_{\mathrm{x}}^{+}$, the whole dispersion law made of the circles periodically arranged are crossed by the line $k_{\mathbf{e}_{\mathbf{k}}}$. Right: for a small positive imaginary part, the poles $k_{p}$ becomes the pair of poles $k_{p}^{ \pm}$with positive or negative imaginary parts. The description of $S_{\mathbf{x}}^{+}$by the direction $\mathbf{e}_{\mathbf{k}}$ leads to the selection of the poles with positive imaginary part.

For the given complex frequency $\omega$ and the unit vector $\mathbf{e}_{\mathbf{k}}$ fixed in $S_{\mathbf{x}}^{+}$, the function $\widetilde{\widetilde{G}}(\mathbf{k}, \omega)$ has a discrete set of poles with respect to the variable $k$, the number of poles could either infinite or zero in the case of a bandgap. Let $k_{p}\left(\omega, \mathbf{e}_{\mathbf{k}}\right)$ be the poles of the modulus $k$ of the wavevector in the direction $\mathbf{e}_{\mathbf{k}}$. These poles correspond to Bloch modes with complex wavevector $k_{p}\left(\omega, \mathbf{e}_{\mathbf{k}}\right) \mathbf{e}_{\mathbf{k}}$ associated to the complex frequency $\omega$ (see figure 10), and can have positive or negative imaginary part since it has been proved in 38 that for non real frequency $\omega$ no real Bloch wavevector exists. Moreover, they form a discrete set which could either infinite or zero, since for real frequency $\omega$, one can prove that the line corresponding to the direction $\mathbf{e}_{\mathbf{k}}$ will intersect the union of the Brillouin zones a periodic number of times if the slope of this line is rational (which is either zero in a stop band or infinite). If the slope 
is irrational these intersection points after $\boldsymbol{K}$-translations to the first Brillouin zone formed a set that is dense in the first Brillouin zone, therefore the number of intersections in that case is again infinite or zero in a bandgap. This still holds by perturbation for frequency $\omega$ with a small imaginary part. Since $\mathbf{e}_{\mathbf{k}} \cdot \mathbf{x}$ is positive, the integral over $k$ can be calculated by closing the line of real numbers by a semi-circle (with infinite radius) in the upper half complex plang 5 . In that case, the set of Bloch wavevectors with positive imaginary part $\mathbf{k}_{p}^{+}\left(\omega, \mathbf{e}_{\mathbf{k}}\right)=k_{p}^{+}\left(\omega, \mathbf{e}_{\mathbf{k}}\right) \mathbf{e}_{\mathbf{k}}$ are picked up by closing the loop in the complex plane:

$$
G(\mathbf{x}, t)=\frac{2 i \pi}{(2 \pi)^{4}} \int_{R_{\eta}} d \omega \exp [-i \omega t] \int_{S_{\mathbf{x}}^{+}} d \mathbf{e}_{\mathbf{k}} \sum_{p} \exp \left[i \mathbf{k}_{p}^{+}\left(\omega, \mathbf{e}_{\mathbf{k}}\right) \cdot \mathbf{x}\right] \operatorname{Res}\left[\mathbf{k}_{p}^{+}\left(\omega, \mathbf{e}_{\mathbf{k}}\right), \omega\right],
$$

where $\operatorname{Res}\left[\mathbf{k}_{p}^{+}\left(\omega, \mathbf{e}_{\mathbf{k}}\right), \omega\right]$ is the residue of $k^{2} \widetilde{\widetilde{G}}\left(k \mathbf{e}_{\mathbf{k}}, \omega\right)$ at the pole $k=k_{p}^{+}(\omega)$. Then the integral over all the directions $\mathbf{e}_{\mathbf{k}}$ in the hemisphere $S_{\mathbf{x}}^{+}$is performed. As a result, all the complex poles $k_{p}^{+}\left(\omega, \mathbf{e}_{\mathbf{k}}\right)$, corresponding to the Bloch wavevectors $\mathbf{k}_{p}^{+}\left(\omega, \mathbf{e}_{\mathbf{k}}\right)$, are picked up and the whole isofrequency dispersion law at the complex frequency $\omega$ is obtained: this isofrequency dispersion law is periodic with respect to the wavevector $\mathbf{k}$. Let $\mathbf{k}_{0}^{+}\left(\omega, \mathbf{e}_{\mathbf{k}}\right)$ be this isofrequency dispersion law restricted to the First Brillouin zone $B$ (see figure 11). First, it is assumed that this isofrequency dispersion law is well-defined for for all direction $\mathbf{e}_{\mathbf{k}}$, i.e. that the function $\mathbf{k}_{0}^{+}\left(\omega, \mathbf{e}_{\mathbf{k}}\right)$ exists for all direction $\mathbf{e}_{\mathbf{k}}$ in the unit sphere $S$. This assumption is correct for frequencies $\omega$ small enough at which, according to homogenization theory 74 , the photonic crystal behaves like a homogeneous medium. Also, without loss of generality, no more than a single mode is assumed in each direction in the first Brillouin zone: in practice, a finite sum over several modes in the first Brillouin could be considered.

Since the dispersion law $\mathbf{k}(\omega)$ is $L^{*}$-periodic, the integral over $S_{\mathbf{x}}^{+}$and the sum over $p$ in the expression $(56)$ can be re-arranged as a periodic sum over the reciprocal lattice $L^{*}$ and the unit sphere $S$ :

$$
\begin{aligned}
\int_{S_{\mathbf{x}}^{+}} d \mathbf{e}_{\mathbf{k}} \sum_{p} \exp \left[i k_{p}^{+}(\omega) \mathbf{e}_{\mathbf{k}} \cdot \mathbf{x}\right] \operatorname{Res}\left[k_{p}^{+}(\omega) \mathbf{e}_{\mathbf{k}}, \omega\right] \\
=\sum_{\boldsymbol{K} \in L^{*}} \int_{S} d \mathbf{e}_{\mathbf{k}} \exp \left[i\left\{\mathbf{k}_{0}^{+}\left(\omega, \mathbf{e}_{\mathbf{k}}\right)+\boldsymbol{K}\right\} \cdot \mathbf{x}\right] \operatorname{Res}\left[\mathbf{k}_{0}^{+}\left(\omega, \mathbf{e}_{\mathbf{k}}\right)+\boldsymbol{K}, \omega\right] .
\end{aligned}
$$

Let the function $R_{\#}\left[\mathbf{x}, k_{0}^{+}\left(\omega, \mathbf{e}_{\mathbf{k}}\right), \omega\right]$ be defined by

$$
R_{\#}\left[\mathbf{x}, \mathbf{k}_{0}^{+}\left(\omega, \mathbf{e}_{\mathbf{k}}\right), \omega\right]=\frac{i}{(2 \pi)^{3}} \sum_{\boldsymbol{K} \in L^{*}} \exp \left[i\left\{\mathbf{k}_{0}^{+}\left(\omega, \mathbf{e}_{\mathbf{k}}\right)+\boldsymbol{K}\right\} \cdot \mathbf{x}\right] \operatorname{Res}\left[\mathbf{k}_{0}^{+}\left(\omega, \mathbf{e}_{\mathbf{k}}\right)+\boldsymbol{K}, \omega\right]
$$

This function has some properties of a Floquet-Bloch component: it is $L^{*}$-periodic with respect to $\mathbf{k}_{0}^{+}\left(\omega, \mathbf{e}_{\mathbf{k}}\right)$ and it satisfies the Bloch boundary conditions with respect to $\mathbf{x}$. With this notation, the expression 56 of $G(\mathbf{x}, t)$ becomes

$$
G(\mathbf{x}, t)=\int_{R_{\eta}} d \omega \exp [-i \omega t] \int_{S} d \mathbf{e}_{\mathbf{k}} R_{\#}\left[\mathbf{x}, \mathbf{k}_{0}^{+}\left(\omega, \mathbf{e}_{\mathbf{k}}\right), \omega\right] .
$$

Now, from the analyticity property 48 , the function $R_{\#}\left[\mathbf{x}, \mathbf{k}_{0}^{+}\left(\omega, \mathbf{e}_{\mathbf{k}}\right), \omega\right]$ must be analytic with respect to $\omega$ in the domain $\operatorname{Im}(\omega)>0$ as soon as the expression above is valid. This suggests that the "dispersion law" $\mathbf{k}_{0}^{+}\left(\omega, \mathbf{e}_{\mathbf{k}}\right)$ could be also analytic under the same conditions if the function $R_{\#}\left[\mathbf{x}, \mathbf{k}_{0}^{+}\left(\omega, \mathbf{e}_{\mathbf{k}}\right), \omega\right]$ could be "inverted". In this aim, the Bloch boundary condition is used: for $\mathbf{a}$ in the lattice $L$ of the photonic crystal, the expression (58) implies

$$
R_{\#}\left[\mathbf{x}+\mathbf{a}, \mathbf{k}_{0}^{+}\left(\omega, \mathbf{e}_{\mathbf{k}}\right), \omega\right]=\exp \left[i \mathbf{k}_{0}^{+}\left(\omega, \mathbf{e}_{\mathbf{k}}\right) \cdot \mathbf{a}\right] R_{\#}\left[\mathbf{x}, \mathbf{k}_{0}^{+}\left(\omega, \mathbf{e}_{\mathbf{k}}\right), \omega\right] .
$$

Hence the function $\exp \left[i \mathbf{k}_{0}^{+}\left(\omega, \mathbf{e}_{\mathbf{k}}\right) \cdot \mathbf{a}\right]$ is analytic as soon as $R_{\#}\left[\mathbf{x}, \mathbf{k}_{0}^{+}\left(\omega, \mathbf{e}_{\mathbf{k}}\right), \omega\right]$ does not vanish. Now, for $\mathbf{k}_{0}^{+}\left(\omega, \mathbf{e}_{\mathbf{k}}\right)$ in the first Brillouin zone, the function $\exp \left[i \mathbf{k}_{0}^{+}\left(\omega, \mathbf{e}_{\mathbf{k}}\right) \cdot \mathbf{a}\right]$ can be

\footnotetext{
${ }^{5}$ Notice that the Fourier transform of the source 41 is analytic of $\mathbf{k}$, since the support of the source is included in the ball of radius $c t_{0}$, and its exponential behavior is exp[ $\left[i k c t_{0}\right]$.
} 
uniquely inverted and thus it is reasonable to consider that the "dispersion law" $\mathbf{k}_{0}^{+}\left(\omega, \mathbf{e}_{\mathbf{k}}\right)$ is analytic with respect to $\omega$. However, it is stressed that all these arguments are valid under the following conditions: the function $\mathbf{k}_{0}^{+}\left(\omega, \mathbf{e}_{\mathbf{k}}\right)$ must exist for all direction $\mathbf{e}_{\mathbf{k}}$ in the unit sphere $S$ and must remain in the First Brillouin zone $B$. These conditions are met for frequencies $\omega$ small enough.
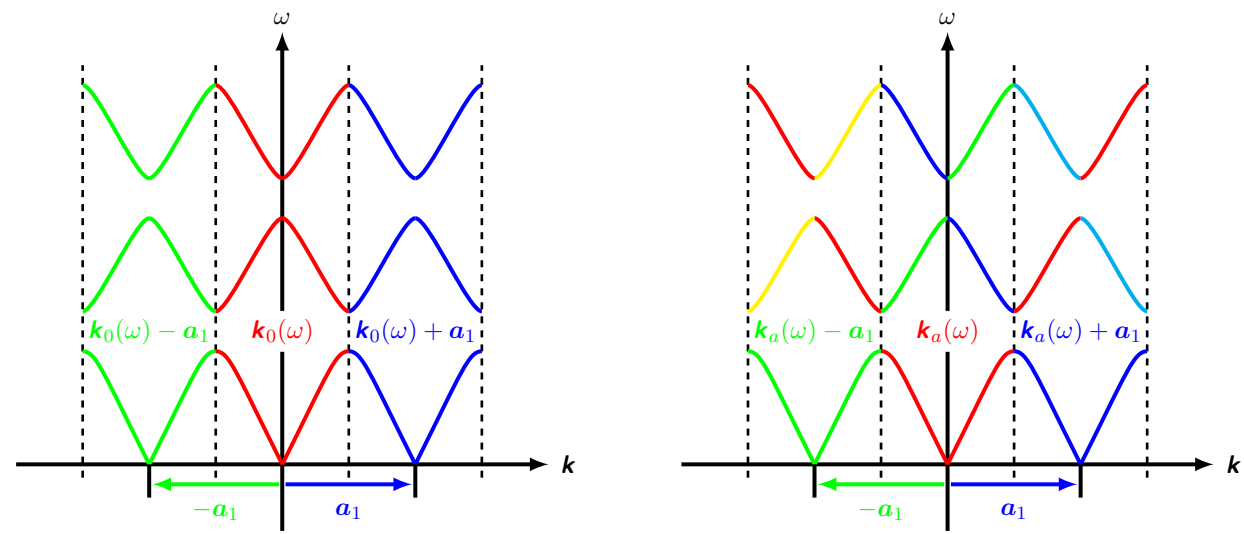

Figure 11: A representation of the periodic dispersion law for real frequencies as the function $\mathbf{k}(\omega)$. Left: the folded dispersion law $\mathbf{k}_{0}(\omega)$ restricted to the First Brillouin zone is represented in red. This curve presents a lack of analyticity at the boundaries of the first Brillouin zone. The whole periodic dispersion law is obtain by translating $\mathbf{k}_{0}(\omega)$ by all the vectors $\mathbf{a}$ in the lattice $L$. The folded parts $\mathbf{k}_{0}(\omega)+\mathbf{a}_{1}$ and $\mathbf{k}_{0}(\omega)-\mathbf{a}_{1}$ are respectively represented in blue and green. Right: the unfolded dispersion law $\mathbf{k}_{a}(\omega)$ is drawn in red and the parts $\mathbf{k}_{a}(\omega)+\mathbf{a}_{1}$ and $\mathbf{k}_{a}(\omega)-\mathbf{a}_{1}$ are respectively represented in blue and green. The unfolded curve $\mathbf{k}_{a}(\omega)$ is not analytic at the boundaries of the Brillouin zones since it is represented in the case of real frequency $\omega$. This unfolded curve $\mathbf{k}_{a}(\omega)$ becomes analytic for frequency $\omega$ with positive imaginary part.

The arguments for the analyticity of $\mathbf{k}_{0}^{+}\left(\omega, \mathbf{e}_{\mathbf{k}}\right)$ presented above seem to fail when $\mathbf{k}_{0}^{+}\left(\omega, \mathbf{e}_{\mathbf{k}}\right)$ reaches the contour of the first Brillouin zone. This is not surprising since, by construction, $\mathbf{k}_{0}^{+}\left(\omega, \mathbf{e}_{\mathbf{k}}\right)$ corresponds to the isofrequency in the First Brillouin zone, and thus results from a folding of the dispersion law (see figure 11). However, the dispersion law $\mathbf{k}_{0}^{+}\left(\omega, \mathbf{e}_{\mathbf{k}}\right)$ can be "unfolded" and the function (58) can expressed as

$$
\begin{aligned}
R_{\#}\left[\mathbf{x}, \mathbf{k}_{0}^{+}\left(\omega, \mathbf{e}_{\mathbf{k}}\right), \omega\right] & =\frac{i}{(2 \pi)^{3}} \sum_{\boldsymbol{K} \in L^{*}} \exp \left[i\left\{\mathbf{k}_{a}^{+}\left(\omega, \mathbf{e}_{\mathbf{k}}\right)+\boldsymbol{K}\right\} \cdot \mathbf{x}\right] \operatorname{Res}\left[\mathbf{k}_{a}^{+}\left(\omega, \mathbf{e}_{\mathbf{k}}\right)+\boldsymbol{K}, \omega\right] \\
& =R_{\#}\left[\mathbf{x}, \mathbf{k}_{a}^{+}\left(\omega, \mathbf{e}_{\mathbf{k}}\right), \omega\right]
\end{aligned}
$$

where the function $\mathbf{k}_{a}^{+}\left(\omega, \mathbf{e}_{\mathbf{k}}\right)$ is the unfolded dispersion law (see figure 11). This expression (61) is just a re-arrangement of the series in 58 since the unfolded dispersion law $\mathbf{k}_{a}^{+}\left(\omega, \mathbf{e}_{\mathbf{k}}\right)$ is defined for high frequencies as a translation of dispersion law in the first Brillouin zone by a vector in the reciprocal lattice $L^{*}$ : for all frequency $\omega$, there exists a vector $\boldsymbol{K}$ in $L^{*}$ such that

$$
\mathbf{k}_{a}^{+}\left(\omega, \mathbf{e}_{\mathbf{k}}\right)=\mathbf{k}_{0}^{+}\left(\omega, \mathbf{e}_{\mathbf{k}}\right)+\boldsymbol{K} .
$$

This unfolded dispersion law can uniquely defined by analytic continuation at the boundaries of the Brillouin zones.

Now, the solution of Maxwell's equations can be expressed using the unfolded dispersion law:

$$
G(\mathbf{x}, t)=\int_{R_{\eta}} d \omega \exp [-i \omega t] \int_{S} d \mathbf{e}_{\mathbf{k}} R_{\#}\left[\mathbf{x}, \mathbf{k}_{a}^{+}\left(\omega, \mathbf{e}_{\mathbf{k}}\right), \omega\right] .
$$

Again, the function $R_{\#}\left[\mathbf{x}, \mathbf{k}_{a}^{+}\left(\omega, \mathbf{e}_{\mathbf{k}}\right), \omega\right]$ must be analytic in the domain of frequencies $\operatorname{Im}(\omega)>0$. Then, the unfolded dispersion law $\mathbf{k}_{a}^{+}\left(\omega, \mathbf{e}_{\mathbf{k}}\right)$ can be extracted from $R_{\#}\left[\mathbf{x}, \mathbf{k}_{a}^{+}\left(\omega, \mathbf{e}_{\mathbf{k}}\right), \omega\right]$ 
using the argument (60): hence it obtained that

$$
\exp \left[i\left\{\mathbf{k}_{a}^{+}\left(\omega, \mathbf{e}_{\mathbf{k}}\right)+\boldsymbol{K}\right\} \cdot \mathbf{a}\right]=\exp \left[i\left\{\mathbf{k}_{0}^{+}\left(\omega, \mathbf{e}_{\mathbf{k}}\right)+\boldsymbol{K}\right\} \cdot \mathbf{a}\right]
$$

which is consistent with (62), but now the inversion of the exponential function must be done in the way that preserves $\mathbf{k}_{a}^{+}\left(\omega, \mathbf{e}_{\mathbf{k}}\right)$ analytic when it spans the whole reciprocal space. Thus the unfolded dispersion law $\mathbf{k}_{a}^{+}\left(\omega, \mathbf{e}_{\mathbf{k}}\right)$ appears as the analytic continuation from the small frequencies $\omega$ of $\mathbf{k}_{0}^{+}\left(\omega, \mathbf{e}_{\mathbf{k}}\right)$ in the first Brillouin zone.

Discussion. Arguments based on the causality principle have been proposed to support that the unfolded dispersion law $\mathbf{k}^{+}(\omega) \equiv \mathbf{k}_{a}^{+}\left(\omega, \mathbf{e}_{\mathbf{k}}\right)$ is an analytic function of the frequency in the domain of complex frequencies $\omega$ with positive imaginary part. This dispersion law $\mathbf{k}^{+}(\omega)$ has been defined with a positive imaginary part. A similar dispersion law $\mathbf{k}^{-}(\omega)$ with a negative imaginary part could be defined using, instead of the hemisphere $S_{\mathbf{x}}^{+}$defined by (54), the hemisphere

$$
S_{\mathbf{x}}^{-}=\left\{\mathbf{e}_{\mathbf{k}} \in S \mid \mathbf{e}_{\mathbf{k}} \cdot \mathbf{x}<0\right\} .
$$

Indeed, in that case, the step from equation $\sqrt[55]{ }$ to equation $(56)$ is performed by closing the real axis by a semi-circle in the lower half complex plane of number $k$, leading to pick up the poles with negative imaginary parts. As a consequence, it is found that in the domain of frequencies $\omega$ with positive imaginary parts two distinct analytic dispersion laws $\mathbf{k}^{ \pm}(\omega)$ exist, with $\mathbf{k}^{+}(\omega)=-\mathbf{k}^{-}(\omega)$.

For small frequencies, the dispersion law $\mathbf{k}^{ \pm}(\omega)$ is well-defined for all direction $\mathbf{e}_{\mathbf{k}}$ in the unit sphere $S$. By analytic continuation, the dispersion law $\mathbf{k}^{ \pm}(\omega)$ appears to be welldefined for all frequencies $\omega$ and for all directions $\mathbf{e}_{\mathbf{k}}$, which could be considered as surprising. Indeed, for real frequencies and real wavevectors the periodicity of photonic crystal implies the presence of bandgaps and, more frequently, of stop bands (i.e. the absence of Bloch modes for certain directions $\mathbf{e}_{\mathbf{k}}$ ). However, when considered in the complex plane, it appears that one can find a complex wavevector $\mathbf{k}$ for all frequency $\omega$.

The present conclusions have been rigorously proved and numerically checked in the onedimensional case in the reference [75. In particular, it has been shown that the wavenumber $k(\omega)$ is an analytic function with respect to the frequency $\omega$ in the domain $\operatorname{Im}(\omega)>0$ and that its imaginary part cannot vanish (passivity requirement). Here, a similar result has been found since the two analytic unfolded dispersion laws $\mathbf{k}^{ \pm}(\omega)$ have keep the same sign for their imaginary part: hence the wavevectors $\mathbf{k}^{ \pm}(\omega)$ cannot vanish. In the one-dimensional case [75], all these results have been confirmed numerically, for instance by checking the validity of the Kramers-Kronig relations.

It is stressed that the arguments presented in this section remain valid in the case of dispersive and absorptive photonic crystals since it preserves the analytic nature with respect to the frequency.

Finally, the reciprocal dispersion law $\omega(\mathbf{k})$ has not been considered in this last section. Indeed, complex Bloch wavevector cannot be directly introduced with Fourier transform since they imply exponential growing in the integrals. However, from the analytic properties 51 of $\widetilde{G}(\mathbf{k}, \omega)$, it can be expected that a well-defined dispersion law $\omega(\mathbf{k})$ could have analytic properties as soon as $\operatorname{Im}(\mathbf{k}) \neq 0$.

\section{Conclusion}

This chapter has been focused on fundamental definitions and properties of dispersion law and group velocity in photonic crystals, including illustrations with numerical examples. This review has shown that numerous questions need to be investigated in the future. The numerical computation of the dispersion law becomes very challenging when the dispersion and absorption are introduced. The techniques based on the introduction of the auxiliary fields 60, 38, 42 have been developed and numerically implemented 64, 67] and are now the basic tool for the emerging topic of quasi-normal modes in photonics [65, 66. It is stressed that these numerical tools use only partial extension of Maxwell's equations where the 
solely dispersion is removed. A challenging question will be to implement the full extension of Maxwell's equations [60, 38, 42] which is associated to a selfadjoint time-independent operator. In particular such an extension may bring an answer to the open questions of the completeness and the normalization of the quasi-normal mode expansions, as well as the link between the complex resonances of the quasi-normal mode expansions and the real spectrum of the augmented selfadjoint operator. Another open question is the analytic structure of the dispersion law. Simple arguments based on the causality principle have been proposed to support some analyticity properties, but rigorous investigations remain definitely necessary. Also, the numerical calculations of the dispersion law in dispersive and absorptive photonic crystals [67] have shown that the resonances associated with the first Brillouin zone contour $\Gamma X M \Gamma$ do not form the contour of the Bloch spectrum: the presence of resonances outside this closed path formed by the resonances of the contour has been highlighted. These preliminary investigations show the potential richness of the vast field of spectral theory of non-selfadjoint operators. Again, the full extension of dispersive and absorptive Maxwell's equations [60, 38, 42 might be a starting point to explore the spectral theory of non-selfadjoint operators.

The modeling of photonic crystals as effective homogeneous media received a keen interest of the community, which led to important contributions in the homogenization theory. Homogenization is an old subject, which dates back to the work by Lord Rayleigh on quasistatic analysis of periodic non dissipative structures [76]. Physicists and mathematicians have used various approaches to replace a periodic structure by an effective medium in the long-wavelength limit with semi-analytical multipole Rayleigh expansions in the dipole approximations [77, plane wave expansions 78, or asymptotic multiple scale expansions techniques [74, 56], and a variety of variational techniques such as the compensated compactness of Tartar and we refer the reader to the book by Milton for a review of low frequency homogenization theories in the composite community [79]. Interestingly, if one adds further corrections to the usual averaged properties of photonic crystals, which is the consecrated high-order homogenization [75, it is necessary to add effective tensors of magneto-optic coupling and permeability to the usual tensor of effective permittivity in order to accurately describe the effective medium. Another pitfall of classical homogenization in photonic crystals is the effect of the boundaries on effective properties [80, 81, 82, 83. These works touch upon concept of non-locality in homogenization of finite photonic crystals with moderate [81, 82, 83] and high [80] contrast. Frequency dispersion in effective properties of high contrast photonic crystals has been also investigated in 84, 80, 85. In order to extract the unusual effective parameters of photonic crystals and metamaterials at any frequency, one can also use some numerical approaches such as the retrieval method, which amounts to fitting the reflection and transmission coefficients of a given complex medium with those of an effective medium through a numerical optimization procedure [86, 87]. Another popular method to compute the effective properties of a periodic structure is a homogenization technique in which macroscopic fields are determined via averaging of the local fields obtained from a full-wave electromagnetic simulation 88 . In the same vein, the high-frequency homogenization allows to reconstruct dispersion curves and associated Bloch waves through a procedure based on numerical field averaging in a periodic cell at any frequency [54.

Finally, the most recent investigations on the bands in photonic crystals focus on topological insulators. For certain ranges of frequency, these structures behave as insulators in their bulk but allow edge states to propagate along a line defect of the photonic crystal. These edge states as surface waves are transversely localized to the defect. Compared to other insulator structures, the main feature of topological insulators [89, 90, 91, 92] is that the edge states have the particularity to be topologically protected or, in other words, very robust to perturbations of the line defect that do not break the bandgap (as for instance local perturbation of the interface of the defect). Moreover these edge states do not backscatter under such perturbations. Examples of topological insulators are given by graphene [93] or topological graphene [92, 94. Indeed, based on the symmetry of the two dimensional honeycomb structure of the graphene crystal (which is invariant by rotation of angle $2 \pi / 3$ and inversion), one can show that certain couple of dispersion curves (see for graphene [95, 93. and for photonic graphene 94]) degenerate at the vertices of the first Brillouin zone (which 
is here hexagonal) where they cross conically on points referred in the literature as Dirac points. Perturbing the dispersion curves at a Dirac point with a line defect that breaks the $\mathcal{P} \mathcal{T}$ symmetry (i.e. the composition of parity-inversion and time-reversal symmetries) of the crystal allows to open a gap (that could be only a local gap for the case of photonic graphene see 94]). In addition, such a defect ensures the existence of topology protected edge states which are localized in this gap [94, 93].

\section{References}

[1] E. Yablonovitch, Inhibited spontaneous emission in solid-state physics and electronics, Phys. Rev. Lett. 58, 002059, (1987).

[2] J. Sajeev, Strong localization of photons in certain disordered dielectric superlattices, Phys. Rev. Lett. 58, 002486, (1987).

[3] V. P. Bykov, Spontaneous emission in a periodic structure, Soviet Journal of Experimental and Theoretical Physics. 35, 000269, (1972).

[4] C. Kittel, Introduction to Solid State Physics, 6th Edition. (John Wiley \& Sons, 1986).

[5] E. Yablonovitch, T. J. Gmitter, and K. M. Leung, Photonic band structure: the facecentered-cubic case employing nonspherical atoms, Phys. Rev. Lett. 67, 002295, (1991).

[6] W. B. Russel, D. A. Saville, and W. R. Schowalter, Colloidal dispersions. (Cambridge University Press, 1995).

[7] A. van Blaaderen, R. Ruel, and P. Wiltzius, Template-directed colloidal crystallization, Nature. 385, 000321, (1997).

[8] S. Y. Lin, J. G. Fleming, D. L. Hetherington, B. K. Smith, R. Biswas, K. M. Ho, M. M. Sigalas, W. Zubrzycki, S. R. Kurtz, and J. Bur, A three-dimensional photonic crystal operating at infrared wavelengths, Nature. 394, 000351, (1998).

[9] J. G. Fleming and S.-Y. Lin, Three-dimensional photonic crystal with a stop band from 1.35 to $1.95 \mu \mathrm{m}$, Opt. Lett. 64, 000049, (1999).

[10] S. Noda, K. Tomoda, N. Yamamoto, and A. Chutinan, Full three-dimensional photonic bandgap crystal at near-infrared wavelengths, Science. 289, 000604, (2000).

[11] S.-Y. Lin, J. G. Fleming, R. Lin, M. M. Sigalas, R. Biswas, and K. M. Ho, Complete three-dimensional photonic bandgap in a simple cubic structure, J. Opt. Soc. Am. B. 18, 000032, (2001).

[12] A. Blanco, E. Chomski, S. Grabtchak, M. Ibisate, S. John, S. Leonard, C. Lopez, F. Meseguer, H. Miguez, J. P. Mondia, G. A. Ozin, O. Toader, and H. M. van Driel, Large-scale synthesis of a silicon photonic crystal with a complete three-dimensional bandgap near 1.5 micrometres, Nature. 405, 000437, (2000).

[13] Y. A. Vlasov, X.-Z. Bo, J. C. Sturm, and D. J. Norris, On-chip natural assembly of silicon photonic bandgap crystals, Nature. 414, 000289, (2001).

[14] T. F. Krauss, R. M. De La Rue, and S. Brand, Two-dimensional photonic-bandgap structures operating at near-infrared wavelengths, Nature. 383, 000699, (1996).

[15] J. Knight, T. Birks, P. Russell, and D. Atkin, All-silica single-mode optical fiber with photonic crystal cladding, Opt. lett. 21, 001547, (1996).

[16] T. P. White, B. T. Kuhlmey, R. C. McPhedran, D. Maystre, G. Renversez, C. M. de Sterke, and L. Botten, Multipole method for microstructured optical fibers. i. formulation, J. Opt. Soc. Am. B. 19, 002322, (2002). 
[17] B. T. Kuhlmey, T. P. White, G. Renversez, D. Maystre, L. C. Botten, C. M. de Sterke, and R. C. McPhedran, Multipole method for microstructured optical fibers. ii. implementation and results, J. Opt. Soc. Am. B. 19, 002331, (2002).

[18] S. Guenneau, C. G. Poulton, and A. B. Movchan, Oblique propagation of elecromagnetic and elastodynamic waves for an array of cylindrical fibres, Proc. R. Soc. A. 459, 002215, (2003).

[19] A. Nicolet, S. Guenneau, C. Geuzaine, and F. Zolla, Modeling of electromagnetic waves in periodic media with finite elements, J. Comp. Appl. Math. 168, 000321, (2004).

[20] F. Zolla, G. Renversez, A. Nicolet, B. Kuhlmey, S. Guenneau, and D. Felbacq, Foundations of photonic crystal fibres. (Imperial College Press, London, 2005).

[21] C. Weisbuch, H. Benisty, S. Olivier, M. Rattier, C. J. M. Smith, and T. F. Krauss, Advances in photonic crystals, Physica Status Solidi. 221, 000093, (2000).

[22] C. R. B. Jamois, Wehrspohn, L. C. Andreani, C. Hermannd, O. Hess, and U. Gosele, Silicon-based two-dimensional photonic crystal waveguides, Nature. 383, 000699, (1996).

[23] S. Noda, A. Chutinan, and M. Imada, Trapping and emission of photons by a single defect in a photonic bandgap structure, Nature. 407, 000608, (2000).

[24] Y. Akahane, T. Asano, B. S. Song, and S. Noda, High-q photonic nanocavity in a two-dimensional photonic crystal, Nature. 425, 000944, (2003).

[25] S. Enoch, B. Gralak, and G. Tayeb, Enhanced emission with angular confinement from photonic crystals, Appl. Phys. Lett. 81, 001588, (2002).

[26] S. Enoch, G. Tayeb, P. Sabouroux, N. Guérin, and P. Vincent, A metamaterial for directive emission, Phys. Rev. Lett. 89, 213902, (2002).

[27] P. Yeh, Electromagnetic propagation in birefringent layered media, J. Opt. Soc. Am. 69, 000742, (1979).

[28] B. Gralak, S. Enoch, and G. Tayeb, Superprism effects and ebg antenna applications, Chapter 10 in Metamaterials: Physics and Engineering Explorations, Edited by $N$. Engheta and R. W. Ziolkowski, John Wiley and Sons. (2006).

[29] S. Enoch, B. Gralak, and G. Tayeb, The richness of the dispersion relation of electromagnetic bandgap materials, IEEE transactions on antennas and propagation. $\mathbf{5 1}$, 002659, (2003).

[30] B. Gralak, S. Enoch, and G. Tayeb, Anomalous refractive properties of photonic crystals, J. Opt. Soc. Am. A. 17, 001012, (2000).

[31] T. Decoopman, G. Tayeb, S. Enoch, D. Maystre, and B. Gralak, Photonic crystal lens: From negative refraction and negative index to negative permittivity and permeability, Phys. Rev. Lett. 97, 073905, (2006).

[32] W. Śmigaj, B. Gralak, R. Pierre, and G. Tayeb, Antireflection gratings for a photoniccrystal flat lens, Opt. Lett. 341, 003532, (2009).

[33] G. Scherrer, M. Hofman, W. Śmigaj, B. Gralak, X. Mélique, D. Vanbésien, O. Lippens, C. Dumas, B. Cluzel, and F. de Fornel, Interface engineering for improved light transmittance through photonic crystal flat lenses, Appl. Phys. Lett. 97, 071119, (2010).

[34] J. B. Pendry, Negative refraction makes a perfect lens, Phys. Rev. Lett. 85, 003966, (2000). 
[35] D. R. Smith, W. J. Padilla, D. C. Vier, S. C. Nemat-Nasser, and S. Schultz, Composite medium with simultaneously negative permeability and permittivity, Phys. Rev. Lett. 84, 004184, (2000).

[36] D. R. Smith, J. B. Pendry, and M. C. K. Wiltshire, Metamaterials and negative refractive index, Science. 305, 000788, (2004).

[37] J. Joannopoulos, R. Meade, and J. Winn, Photonic crystals. (Princeton University Press, 1995).

[38] A. Tip, A. Moroz, and J.-M. Combes, Band structure of absorptive photonic crystals, J. Phys. A: Mathematical and General. 33, 006223, (2000).

[39] A. Bensoussan, J.-L. Lions, and G. Papanicolaou, Asymptotic analysis for periodic structures. (North-Holland, Amsterdam, 1978).

[40] P. Kuchment, Floquet Theory for Partial Differential Equations. (Birkhuser Verlag, 1993).

[41] M. Reed and B. Simon, Methods of Modern Mathematical Physics. vol. II: Fourier Analysis, Self-Adjointness, (Academic Press, 1975).

[42] B. Gralak and A. Tip, Macroscopic Maxwells equations and negative index materials, J. Math. Phys. 51, 052902, (2010).

[43] B. Gralak, Analytic properties of the electromagnetic Greens function, J. Math. Phys. 58, 071501, (2017).

[44] K. M. Leung and Y. F. Liu, Full vector wave calculation of photonic band structures in face-centered-cubic dielectric media, Phys. Rev. Lett. 65, 002646, (1990).

[45] Z. Zhang and S. Satpathy, Electromagnetic wave propagation in periodic structures: Bloch wave solution of Maxwell's equations, Phys. Rev. Lett. 65, 002650, (1990).

[46] K. Ho, C. Chan, and C. Soukoulis, Existence of photonic gap in periodic dielectric structures, Phys. Rev. Lett. 65, 003152, (1990).

[47] K. M. Ho, C. T. Chan, C. M. Soukoulis, R. Biswas, and M. Sigalas, Photonic band gaps in three dimensions: new layer-by-layer periodic structures, Solid State Communications. 89, 000413, (1994).

[48] A. Chutinan and S. Noda, Effect of structural fluctuations on the photonic bandgap during fabrication of a photonic crystal, J. Opt. Soc. Am. B. 16, 000240, (1999).

[49] V. Lousse, J.-P. Vigneron, X. Bouju, and J.-M. Vigoureux, Atomic radiation rates in photonic crystals, Phys. Rev. B. 64, 201104(R), (2001).

[50] D. M. Whittaker, Inhibited emission in photonic woodpile lattices, Opt. Lett. 25, 000779, (2000).

[51] S. G. Johnson and J. D. Joannopoulos, Block-iterative frequency-domain methods for maxwell's equations in a planewave basis, Opt. Express. 8, 000173, (2001).

[52] comsol multiphysics, www.comsol.fr.

[53] G. Scherrer, M. Hofman, W. Śmigaj, M. Kadic, T.-M. Chang, X. Mélique, D. Lippens, O. Vanbésien, B. Cluzel, F. de Fornel, S. Guenneau, and B. Gralak, Photonic crystal

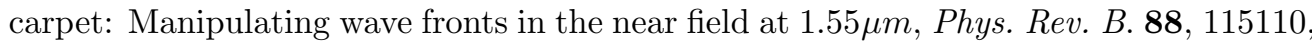
(2013).

[54] R. Craster, J. Kaplunov, and A. Pichugin, High-frequency homogenization for periodic media, Proc. R. Soc. A. p. 20090612, (2010). 
[55] T. Antonakakis, R. Craster, and S. Guenneau, High-frequency homogenization of zerofrequency stop band photonic and phononic crystals, New J. Phys. 15, 103014, (2013).

[56] S. Guenneau and F. Zolla, Homogenization of three-dimensional finite photonic crystals, Progress in Electromagnetics Research. 27, 000091, (2000).

[57] W. Smigaj and G. Gralak, Semianalytical design of antireflection gratings for photonic crystals, Phys. Rev. B. 85, 035114, (2012).

[58] A. Moroz, Three-dimensional complete photonic-bandgap structures in the visible, Phys. Rev. Lett. 83, 005274, (1999).

[59] H. van der Lem and A. Moroz, Towards two-dimensional complete photonic bandgap structures below infrared wavelengths, J. Opt. A: Pure Applied Optics. 2, 000395 , (2000).

[60] A. Tip, Linear absorptive dielectric, Phys. Rev. A. 57, 004818, (1998).

[61] A. Tip, Canonical formalism and quantization for a class of classical fields with application to radiative atomic decay in dielectric, Phys. Rev. A. 56, 005022, (1997).

[62] H. van der Lem, A. Tip, and A. Moroz, Band structure of absorptive two-dimensional photonic crystals, J. Opt. Soc. Am. B. 20, 001334, (2003).

[63] J.-M. Combes, B. Gralak, and A. Tip, Spectral properties of absorptive photonic crystals, Contemporary Mathematics Waves in Periodic and Random Media. 339, 1, (2003).

[64] A. Raman and S. Fan, Photonic band structure of dispersive metamaterials formulated as a hermitian eigenvalue problem, Phys. Rev. Lett. 104, 087401, (2010).

[65] P. Lalanne, W. Yan, K. Vynck, C. Sauvan, and J.-P. Hugonin, Light interaction with photonic and plasmonic resonances, Laser Photonics Rev. 2018, 1700113, (2018).

[66] W. Yan, R. Faggiani, and P. Lalanne, Rigorous modal analysis of plasmonic nanoresonators, Phys. Rev. B. 97, 205422, (2018).

[67] Y. Brûlé, B. Gralak, and G. Demésy, Calculation and analysis of the complex band structure of dispersive and dissipative two-dimensional photonic crystals, J. Opt. Soc. Am. B. 33, 000691, (2016).

[68] C. Geuzaine and J.-F. Remacle, Gmsh: a three-dimensional finite element mesh generator with built-in pre- and post-processing facilities, International Journal for Numerical Methods in Engineering. 79, 001309, (2009).

[69] J. Webb and B. Forgahani, Hierarchal scalar and vector tetrahedra, IEEE Transactions on Magnetics. 29, 001495, (1993).

[70] P. Dular, C. Geuzaine, F. Henrotte, and W. Legros, A general environment for the treatment of discrete problems and its application to the finite element method, IEEE Transactions on Magnetics. 34, 003395, (1998).

[71] V. Hernandez, J. E. Roman, and V. Vidal, SLEPc: A scalable and flexible toolkit for the solution of eigenvalue problems, ACM Transactions on Mathematical Software. 31, 000351, (2005).

[72] H. Knorrer and E. Trubowitz, A directional compactification of the complex bloch variety, Commentarii Mathematici Helvetici. 65, 000114, (1990).

[73] R. Dautray and J.-L. Lions, Mathematical Analysis and Numerical Methods for Science and Technology. Volume 5 Evolution Problems I. (Springer, 2000).

[74] A. Bensoussan, J.-L. Lions, and G. Papanicolaou, Asymptotic analysis for periodic structures. (North-Holland, 1978). 
[75] Y. Liu, S. Guenneau, and B. Gralak, Artificial dispersion via high-order homogenization: magnetoelectric coupling and magnetism from dielectric layers, Proc. R. Soc. A. 469, 20130240, (2013).

[76] L. Rayleigh, On the influence of obstacles arranged in rectangular order upon the properties of a medium, Philosophical Magazine. 34, 481-502, (1892).

[77] R. C. McPhedran, C. Poulton, N. Nicorovici, and A. Movchan, Low frequency corrections to the static effective dielectric constant of a two-dimensional composite material, Proc. R. Soc. A. 452, 002231, (1996).

[78] P. Halevi, A. Krokhin, and J. Arriaga, Photonic crystal optics and homogenization of 2d periodic composites, Phys. Rev. Lett. 82, 000719, (1999).

[79] G. Milton, The theory of composites. (Cambridge University Press, 2002).

[80] M. Silveirinha, Additional boundary condition for the wire medium, IEEE transactions on antennas and propagation. 54, 001766, (2006).

[81] R. Pierre and G. Gralak, Appropriate truncation for photonic crystals, J. Mod. Opt. $\mathbf{5 5}, 001759,(2008)$.

[82] W. Smigaj and B. Gralak, Validity of the effective-medium approximation of photonic crystals, Phys. Rev. B. 77, 235445, (2008).

[83] V. A. Markel and I. Tsukerman, Current-driven homogenization and effective medium parameters for finite samples, Phys. Rev. B. 88, 125131, (2013).

[84] D. Felbacq and G. Bouchitte, Homogenization of a set of parallel fibres, Waves in random media. 7, 000245, (1997).

[85] M. Silveirinha, Metamaterial homogenization approach with application to the characterization of microstructured composites with negative parameters, Phys. Rev. B. 75, 115104, (2007).

[86] T. Koschny, P. Markos, D. R. Smith, and C. M. Soukoulis, Resonant and antiresonant frequency dependence of the effective parameters of metamaterials., Phys. Rev. E. 68, 065602(R), (2003).

[87] C. Menzel, C. Rockstuhl, T. Paul, F. Lederer, and T. Pertsch, Retrieving effective parameters for metamaterials at oblique incidence., Phys. Rev. B. 77, 195328, (2008).

[88] D. Smith and J. Pendry, Homogenization of metamaterials by field averaging, J. Opt. Soc. Am. B. 23, 000391, (2006).

[89] W. J. Chen, S. J. Jiang, X. D. Chen, B. Zhu, L. Zhou, Dong, J. W., and C. T. Chan, Experimental realization of photonic topological insulator in a uniaxial metacrystal waveguide., Nat. Comm. 5, 005782, (2014).

[90] C. L. Kane and E. J. Mele, Z2 topological order and the quantum spin hall effect., Phys. Rev. 14, 146802, (2005).

[91] S. Raghu and F. D. M. Haldane, Analogs of quantum-hall-effect edge states in photonic crystals., Phys. Rev. A. 78, 033834, (2008).

[92] M. C. Rechtsman, J. M. Zeuner, Y. Plotnik, Y. Lumer, D. Podolsky, F. Dreisow, and A. Szameit, Photonic floquet topological insulators., Nature. 496, 000196, (2013).

[93] C. L. Fefferman, J. P. Lee-Thorp, and M. I. Weinstein, Honeycomb schrödinger operators in the strong binding regime, Communications on Pure and Applied Mathematics. 71, 001178, (2018). 
[94] J. P. Lee-Thorp, M. I. Weinstein, and Y. Zhu, Elliptic operators with honeycomb symmetry: Dirac points, edge states and applications to photonic graphene, available on arxiv at https: //arxiv. org/abs/1710.03389. (2017).

[95] P. R. Wallace, The band theory of graphite, Phys. Rev. 71, 000622, (1947). 